TRANSACTIONS OF THE

AMERICAN MATHEMATICAL SOCIETY

Volume 352, Number 12, Pages 5485-5510

S 0002-9947(00)02054-7

Article electronically published on August 8, 2000

\title{
CURVES ON NORMAL SURFACES
}

\author{
GUNNAR FLØYSTAD
}

\begin{abstract}
We study locally Cohen-Macaulay space curves lying on normal surfaces. We prove some theorems on the behaviour of the cohomology functions and initial ideals of such space curves, which give a basic distinction between those curves and curves lying on non-normal surfaces.
\end{abstract}

A natural step in the study and classification of space curves is to classify the Hilbert functions of space curves, or, more generally, cohomology functions of space curves. A closely related approach is to study the somewhat more refined invariants, the generic initial ideal and higher initial ideal, and classify which of them can occur for smooth, integral or locally Cohen-Macaulay space curves.

Given a space curve $C$ on a surface $S \subseteq \mathbf{P}^{3}$, one may do descending biliaison on the surface $S$ and end up with a curve $X$ minimal in its biliaison class on the surface $S$. On the other hand, one might consider a surface $T$ of minimal degree such that $C$ may be linked $(S, T)$ to a curve $Y$.

In these cases it will often happen that $X$ or $Y$ will have non-reduced components. The invariants of $C$ may be deduced from the invariants of $X$ or $Y$. Hence it is natural to try to get an understanding of the invariants of curves on $S$ with nonreduced components.

Here it turns out that there is a basic distinction between when $S$ is normal and when $S$ is not normal. The reason is that if one has a reduced curve $D$ on $S$ then a multiplicity $n$ structure on $D$ in $S$ is uniquely determined if $S$ is normal, while if $S$ is not normal there may exist many (in fact infinitely many) multiplicity $n$ structures on $D$ in $S$.

For $C$ a curve on a surface $S$ of degree $s$ there is a basic sequence

$$
0 \longrightarrow \mathcal{L} \longrightarrow \mathcal{O}_{S} \longrightarrow \mathcal{O}_{C} \longrightarrow 0,
$$

where $\mathcal{L}$ is the ideal sheaf of $C$ in $S$. This also gives rise to a sequence

$$
0 \longrightarrow \mathcal{O}_{S}(s-4) \longrightarrow \mathcal{L}^{\vee}(s-4) \longrightarrow \omega_{C} \longrightarrow 0 .
$$

In both cases $\mathcal{L}$ and $\mathcal{L}^{\vee}(s-4)$ are torsion free rank one sheaves on $S$ with local

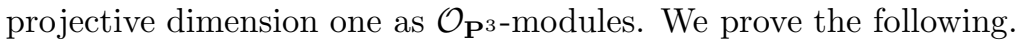

Theorem 2.1. Let $S \subseteq \mathbf{P}^{3}$ be a normal surface of degree $s$, and $\mathcal{L}$ a torsion free rank one sheaf on $S$ with local projective dimension one as $\mathcal{O}_{\mathbf{P}^{3}}$-module. Let the minimal generators of $H_{*}^{0} \mathcal{L}=\bigoplus_{n \in \mathbf{Z}} H^{0} \mathcal{L}(n)$ occur in degrees $n_{0} \leq n_{1} \leq \cdots \leq n_{r}$. Then $n_{j}-n_{j-1} \leq s-2$ for $j=1, \ldots, r$, and the regularity of $\mathcal{L}$ is $\leq n_{r}+(s-2)$.

Received by the editors July 4, 1996 and, in revised form, September 22, 1997.

2000 Mathematics Subject Classification. Primary 14H50.

Key words and phrases. Space curves, normal surfaces, initial ideals.

(C)2000 American Mathematical Society 
The theorem is sharp. It is not true if $S$ is not normal. In fact, on a given nonnormal surface $S$ there may exist $\mathcal{L}$ on $S$ with a gap between successive degrees of minimal generators of $H_{*}^{0} \mathcal{L}$ exceeding any given number.

Of course, by the sequences (11) and (2) this gives immediate consequences for the generators of the homogeneous ideal $I_{C}$ and the speciality module $H_{*}^{0} \omega_{C}$.

The proof of the theorem is an elaboration of the following key idea. Let $D$ be a smooth irreducible curve on a smooth surface $S$. Let $D^{(i)}$ be the multiple $i$ structure on $D$ in $S$. Then there are sequences

$$
0 \longrightarrow \mathcal{F}_{i-1} \longrightarrow \mathcal{O}_{D^{(i)}} \longrightarrow \mathcal{O}_{D^{(i-1)}} \longrightarrow 0
$$

where the line bundles $\mathcal{F}_{i}$ are related by

$$
\mathcal{F}_{i}=\mathcal{F}_{i-1} \otimes \omega_{D}^{\vee}(s-4) .
$$

If $\mathcal{O}_{D^{(n)}}(-r)$ has sections for some $-r<0$, then $\mathcal{F}_{i}(-r)$ also has sections for some $i \leq n-1$. Since $\mathcal{F}_{0}=\mathcal{O}_{D}$, the line bundle $\omega_{D}^{\vee}(s-4)$ must have positive degree. Thus the $\mathcal{F}_{i}$ have successively more and more positive degree. However they can not increase too fast in degree. Since

$$
\operatorname{deg} \omega_{D}^{\vee}(s-4) \leq \operatorname{deg} \mathcal{O}_{D}(s-2)
$$

(with equality if and only if $D$ is a line), we have

$$
\operatorname{deg} \mathcal{F}_{i} \leq \operatorname{deg} \mathcal{F}_{i-1}(s-2)
$$

which is the reason for the number $s-2$.

When it comes to the Hilbert function of the ideal $I_{C}$, it turns out that Theorem 2.1 is most conveniently interpreted in terms of the generic initial ideal for the reverse lexicographic order.

Note. Throughout the paper, whenever we consider a curve $C$ and its initial ideal or higher initial ideal, we always assume the curve to be given in general coordinates. Thus these are the generic initial ideal and generic higher initial ideal for the reverse lexicographic order.

An almost immediate consequence of Theorem 2.1 is

Theorem 4.1. Let

$$
\operatorname{in}\left(I_{C}\right)=\left(\mathbf{x}^{I_{0}}, \mathbf{x}^{I_{1}}, \ldots, \mathbf{x}^{I_{t}}\right)
$$

be a minimal generating set. Consider the set

$$
\left\{\mathbf{x}^{I_{0}}, \ldots, \mathbf{x}^{I_{t}}\right\} \backslash\left\{x_{0}^{s}\right\} .
$$

Let the elements of this set have degrees

$$
n_{0} \leq n_{1} \leq \cdots \leq n_{t^{\prime}} .
$$

Then $n_{j}-n_{j-1} \leq s-2$ for $j=1, \ldots, t^{\prime}$.

In other words, the gaps between the degrees of the minimal generators of the initial ideal, excluding $x_{0}^{s}$ if that is a minimal generator, are at most $s-2$.

The theorem is sharp. It is not true if $S$ is a fixed non-normal surface. Then, given any number, there may exist smooth curves on $S$ with a gap between successive degrees of minimal generators of $\operatorname{in}\left(I_{C}\right)$ (none of which are $x_{0}^{s}$ if that is a minimal generator) exceeding this number. 
There is also a corresponding result for the higher initial ideal $\operatorname{in}_{1}\left(I_{C}\right)$ (Theorem $4.2)$.

We give some examples. For the diagram notation given here we refer to $\mathrm{Fl}-\mathrm{Gr}$. or [F], section 13 .

Example 1. Suppose $S$ is a normal quadric surface (i.e. $S$ is reduced and irreducible). Excluding $x_{0}^{2}$ there can be no gaps between the degrees of the generators of in $\left(I_{C}\right)$. Thus the initial ideal must look like the following :

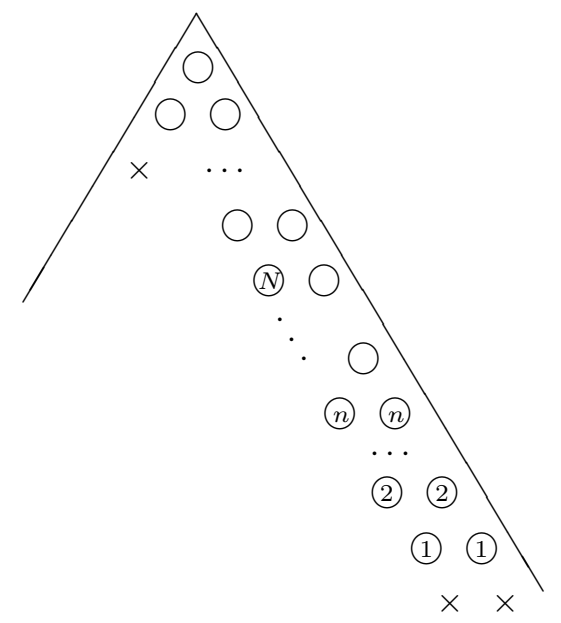

It is not difficult to work out that one must have $N=n$ or $n+1$. This may for instance be seen from the fact that a curve in $C+r H$ for $r \gg 0$ must have connected numerical character.

Example 2. Suppose $S$ is a normal cubic surface. The following monomial ideal has generators in degree 5 and 7 but none in degree 6 . Thus it cannot be the initial ideal of a curve on $S$.

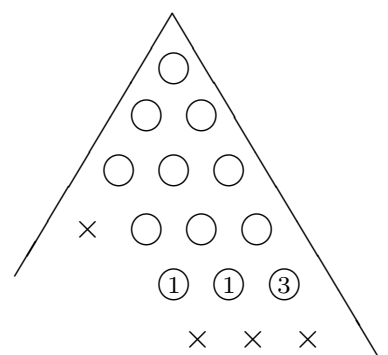

It turns out that the most convenient interpretation of these results for cohomology functions of $C$ is in terms of the Rao function

$$
\rho(n)=h^{1} \mathcal{I}_{C}(n) .
$$


Let $\Delta^{2} \rho$ be the second difference function. Let $t$ be minimal such that $C$ is in a complete intersection $(s, t)$. Consider the interval $A=[a, a+(s-3)]$, where $a \geq t+s-1$. A consequence of Theorem 2.1 is the following: If $\Delta^{2} \rho(n)=0$ for $n \in A$, then $\Delta^{2} \rho(n)=0$ for $n \geq a$. Actually a closer investigation shows that something more can be deduced. Suppose $C$ is on a normal surface $S$ with $s=3$. Let $e=e_{C}$ be the speciality index and let $\delta_{a}(n)=\delta_{a n}$ be the Kronecker delta function.

Theorem $3.2(s=3)$. The function $\Delta^{2} \rho(n)+\delta_{e-(s-4)}(n)$ is weakly increasing in the range $n \leq e-(s-4)$.

Theorem $3.3(s=3)$. The function $\Delta^{2} \rho(n)+\delta_{t+s-2}(n)$ is weakly decreasing in the range $n \geq t+s-2$.

The method used to prove these theorems is not particular to cubic surfaces. For a normal surface of degree $s \geq 4$ it has been awkward to find a precise formulation for what should hold, and probably still more awkward to prove it, but a statement should be something along these lines: the function

$$
\tau(n)=\frac{1}{s-2}\left(\sum_{i \in[n, \ldots, n+(s-3)]} \Delta^{2} \rho(i)\right)
$$

is weakly increasing in the rang $n+(s-3) \leq e-(s-4)$ (resp. decreasing for $n \geq t+s-2$ ). (This form of statement is probably not true, but it gives the intuitive idea.)

We also interpret Theorems 3.2 and 3.3 in terms of initial ideals. Let $l(\nu)$ be the number of generators of in $\left(I_{C}\right)$ of degree $\nu$ which are divisible by $x_{2}$.

Theorem $5.1(s=3)$. The function $l(\nu)+\delta_{t+s-2}(\nu)$ is weakly decreasing in the range $\nu \geq t+s-2$.

There is also a corresponding theorem for the higher initial ideal (Theorem 5.2).

Example 3. The following monomial ideal cannot be the initial ideal of a curve on a normal cubic surface:

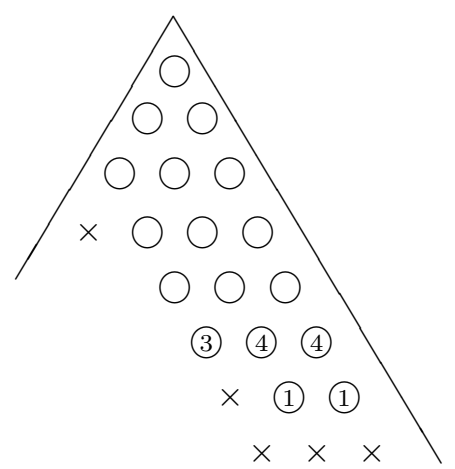

We apply Theorem 5.1 with $t=6$. The number of minimal generators divisible by $x_{2}$ is 1 in degree $t+2$ and 2 in degree $t+3$. This is impossible by Theorem 5.1 . But maybe it could be the initial ideal of a curve on a non-normal cubic surface. 
The reason we put emphasis on the initial ideal interpretation of these results is that we see the problem of understanding what initial ideals can occur for space curves as a natural question in the classification of space curves. And moreover, and not least important, it was by considering initial ideals that we were first led to Theorem 2.1 and subsequently to the other theorems of this paper.

The outline of the paper is as follows. Section 1 contains some preliminary results and notation. Section 6 is the main technical section of this paper. It studies non-reduced curves on normal surfaces. In Sections 2, 3, 4, and 5 we derive the theorems, and in a way they are all corollaries to Section 6 .

In Section 2 we prove Theorem 2.1. In Section 3 we prove Theorems 3.2 and 3.3. Section 4 gives the initial ideal interpretation of Theorem 2.1. Section 5 gives the initial ideal interpretation of 3.2 and 3.3 .

In Section 7 we show by an example what may happen on a non-normal surface $S$. We consider the irreducible non-normal cubic surface. Then, given any number, we give examples of smooth curves $C$ on $S$ with a gap between successive degrees of minimal generators of $I_{C}$ or $\operatorname{in}\left(I_{C}\right)$ exceeding this number.

Throughout the paper all curves are assumed to be locally Cohen-Macaulay unless stronger conditions are explicitly stated.

\section{Preliminaries}

In this section we collect some notation and general facts used in this paper.

Sheaves of local depth one on a curve. Let $X$ be a locally Cohen-Macaulay curve, i.e., a curve without isolated or embedded points. Let $\mathcal{M}$ be a coherent sheaf on $X$. We denote by $\mathcal{M}^{\circ}$ the quotient sheaf of $\mathcal{M}$ obtained by dividing out by the intersection of the primary submodules primary to the minimal prime ideals of $\mathcal{M}$. More intuitively, $\mathcal{M}^{\circ}$ is obtained from $\mathcal{M}$ by throwing away all module components of dimension 0 . Note that $\mathcal{M}^{\circ}$ will have local depth one.

Suppose now that $\mathcal{R} \subseteq \mathcal{M}$ is an inclusion of coherent sheaves of local depth one on $X$. We may form the quotient $\mathcal{A}=\mathcal{M} / \mathcal{R}$. This gives a composition

$$
\mathcal{M} \longrightarrow \mathcal{A} \longrightarrow \mathcal{A}^{\circ}
$$

We denote by $\hat{\mathcal{R}}$ the kernel of this composition.

Dualizing functors. Suppose now that $X$ is a closed subscheme of a locally CohenMacaulay scheme $Y$ of pure dimension $r$. Suppose $Y$ has a canonical sheaf $\omega_{Y}$. Then $X$ has a canonical sheaf $\omega_{X}=\mathcal{E} x t_{\mathcal{O}_{Y}}^{r-1}\left(\mathcal{O}_{X}, \omega_{Y}\right)$ by [Ei], Theorem 2.15. In the following we consider the category of coherent sheaves of local depth one on $X$ and let $\mathcal{M}$ be an object in this category.

Proposition 1.1. $\quad$ 1. $\mathcal{H}^{\circ} m_{\mathcal{O}_{X}}\left(-, \omega_{X}\right)$ is a dualizing functor on $X$; in particular, the natural map

$$
\mathcal{M} \longrightarrow \mathcal{H}^{\prime o m} \mathcal{O}_{X}\left(\mathcal{H o m}_{\mathcal{O}_{X}}\left(\mathcal{M}, \omega_{X}\right), \omega_{X}\right)
$$

is an isomorphism.

2. $\mathcal{E}_{x} t_{\mathcal{O}_{Y}}^{i}\left(\mathcal{M}, \omega_{Y}\right)=0$ for $i \neq r-1$.

3. There is an isomorphism of functors

$$
\mathcal{H o m}_{\mathcal{O}_{X}}\left(-, \omega_{X}\right) \cong \mathcal{E}_{x} t_{\mathcal{O}_{Y}}^{r-1}\left(-, \omega_{Y}\right) .
$$


Proof. 1. This is [Ei], Theorem $21.21 \mathrm{c}$.

3. Given 2. above, the proof of $\mathrm{Ha}$, III, Lemma 7.4, goes through.

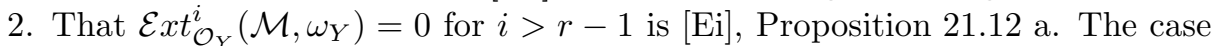
when $i<r-1$ comes from the following.

Lemma 1.2. Let $A$ be a local Cohen-Macaulay ring of dimension $d$.

a) Then $\omega_{A}$ is a maximal Cohen-Macaulay module, i.e., depth $\omega_{A}=d$.

b) If $M$ is a maximal Cohen-Macaulay module and $N$ has support in dimension $\leq e$, then $\operatorname{Ext}_{A}^{i}(N, M)=0$ for $i<d-e$.

Proof. a) This is [Ei], Proposition 21.8.

b) This follows by [Ei], Propositions 18.4 and 18.3.

This also finishes the proof of Proposition 1.1.

Residual schemes. Now suppose that the curve $X$ is on a normal surface $S$. Given a subscheme $D$ of $X$, we may define the residual scheme $Y$ of $D$ in $X$ by

$$
\mathcal{I}_{Y / X}=\left(0: \mathcal{O}_{X} \mathcal{I}_{D / X}\right) \text {. }
$$

By the proof of [Ei], Theorem 21.23, $Y$ is a locally Cohen-Macaulay subcurve of $X$. This gives a sequence

$$
0 \longrightarrow \mathcal{I}_{Y / X} \longrightarrow \mathcal{O}_{X} \longrightarrow \mathcal{O}_{Y} \longrightarrow 0 \text {. }
$$

We claim that $\mathcal{I}_{Y / X}$ is an invertible sheaf on $D$ outside the singularities of $S$. Clearly, since $Y$ is a Cartier divisor outside $\operatorname{Sing} S$, the sheaf $\mathcal{I}_{Y / X}$ is invertible on its support outside Sing $S$. Since $\operatorname{Ann} \mathcal{I}_{Y / X} \supseteq \mathcal{I}_{D / X}$, the support is contained in $D$, but by Hilbert polynomial considerations we must have equality, so the support of $\mathcal{I}_{Y / X}$ is $D$.

Rank one sheaves on a surface. Now on the surface $S$ there is an exact sequence

$$
0 \longrightarrow \mathcal{L} \longrightarrow \mathcal{O}_{S} \longrightarrow \mathcal{O}_{X} \longrightarrow 0,
$$

where $\mathcal{L}=\mathcal{I}_{X / S}$. Here $\mathcal{L}$ is a rank 1 sheaf on $S$ of local projective dimension one as

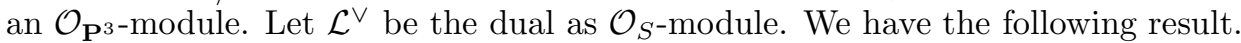

Lemma 1.3. Suppose the local projective dimension $\operatorname{lpd}_{\mathcal{O}_{\mathbf{P}^{3}}} \mathcal{L}=1$. Then:

1. $\operatorname{lpd}_{\mathcal{O}_{\mathbf{P}^{3}}} \mathcal{L}^{\vee}=1$.

2. $\mathcal{L}^{\vee \vee}=\mathcal{L}$.

Proof. There is a sequence

$$
0 \longrightarrow \mathcal{G} \longrightarrow \mathcal{H} \longrightarrow \mathcal{L} \longrightarrow 0
$$

where $\mathcal{G}$ and $\mathcal{H}$ are locally free on $\mathbf{P}^{3}$. This gives

$$
0 \longrightarrow \mathcal{H}^{\vee} \longrightarrow \mathcal{G}^{\vee} \longrightarrow \mathcal{E} x t^{1}\left(\mathcal{L}, \mathcal{O}_{\mathbf{P}^{3}}\right) \longrightarrow 0,
$$

where ${ }^{\vee}$ denotes the dual as $\mathcal{O}_{\mathbf{P}^{3} \text {-module. Since }}$

$$
\mathcal{E} x t^{1}\left(\mathcal{L}, \mathcal{O}_{\mathbf{P}^{3}}\right)=\mathcal{H} \operatorname{om}\left(\mathcal{L}, \mathcal{O}_{S}(s)\right)=\mathcal{L}^{\vee}(s),
$$

we get $\operatorname{lpd}_{\mathcal{O}_{\mathbf{P}^{3}}} \mathcal{L}^{\vee}=1$. Dualizing once more gives

$$
0 \longrightarrow \mathcal{G} \longrightarrow \mathcal{H} \longrightarrow \mathcal{E} x t^{1}\left(\mathcal{L}^{\vee}(s), \mathcal{O}_{\mathbf{P}^{3}}\right) \longrightarrow 0
$$


Here

$$
\mathcal{E} x t^{1}\left(\mathcal{L}^{\vee}(s), \mathcal{O}_{\mathbf{P}^{3}}\right)=\mathcal{H o m}\left(\mathcal{L}^{\vee}, \mathcal{O}_{S}\right)=\mathcal{L}^{\vee \vee}
$$

so we get $\mathcal{L}=\mathcal{L}^{\vee \vee}$.

\section{The MAIN RESUlT}

Let $S \subseteq \mathbf{P}^{3}$ be a normal surface (i.e., with a finite number of singularities). Let $C \subseteq S$ be a locally Cohen-Macaulay curve. There is an exact sequence

$$
0 \longrightarrow \mathcal{L} \longrightarrow \mathcal{O}_{S} \longrightarrow \mathcal{O}_{C} \longrightarrow 0,
$$

where $\mathcal{L}=\mathcal{I}_{C / S}$. Here $\mathcal{L}$ is a rank 1 sheaf on $S$ of local projective dimension one as an $\mathcal{O}_{\mathbf{P}^{3}}$-module. Let $\mathcal{L}^{\vee}$ be the dual as an $\mathcal{O}_{S}$-module. Then there is a sequence

$$
0 \longrightarrow \mathcal{O}_{S}(s-4) \longrightarrow \mathcal{L}^{\vee}(s-4) \longrightarrow \omega_{C} \longrightarrow 0,
$$

and furthermore a sequence

$$
0 \longrightarrow \mathcal{O}_{\mathbf{P}^{3}}(-s) \longrightarrow \mathcal{I}_{C / \mathbf{P}^{3}} \longrightarrow \mathcal{L} \longrightarrow 0
$$

The following theorem then says something about the generators of the homogeneous ideal of the curve, and also something about the generators of the graded module $H_{*}^{0} \omega_{C}$.

Theorem 2.1. Let $S \subseteq \mathbf{P}^{3}$ be a normal surface of degree $s$ and let $\mathcal{L}$ be a rank 1 sheaf on $S$ with $\operatorname{lpd}_{\mathcal{O}_{\mathbf{P}^{3}}} \mathcal{L}=1$. Let the minimal set of generators of $H_{*}^{0} \mathcal{L}$ occur in degrees

$$
n_{0} \leq n_{1} \leq \cdots \leq n_{r}
$$

Then

$$
n_{j}-n_{j-1} \leq s-2
$$

for $j=1, \ldots, r$, and the regularity of $\mathcal{L}$ is $\leq n_{r}+(s-2)$.

Remark. Looking at the homogeneous ideal $I_{C} \subseteq k\left[x_{0}, x_{1}, x_{2}, x_{3}\right]$, this implies that the gaps in the successive degrees of the generators of $I_{C}$ (excluding the form defining the surface $S$, if that is a minimal generator) are at most $s-2$.

Furthermore one sees that if the sequence (3) is obtained by taking a section of $H_{*}^{0} \mathcal{L}^{\vee}$ of minimal degree (which is equivalent to $e_{C} \leq s-4$ ), then the gaps between successive degrees of generators of $H_{*}^{0} \omega_{C}$ are at most $s-2$.

Remark. The theorem is sharp. Let $S$ be a smooth surface with a line $L$ and let $L^{(n)}$ be a multiple $n$ structure on $L$. If $C$ is linked to $L^{(n)}$ on $S$ and $\mathcal{L}$ is the ideal sheaf of $C$, then all gaps between minimal generators of $H_{*}^{0} \mathcal{L}$ of successive degrees are of size $s-2$.

Remark. This theorem does not hold for curves on non-normal surfaces. In fact, if $S$ is a given non-normal surface, then given any number, there may exist $\mathcal{L}$ with a gap between successive degrees of generators of $H_{*}^{0} \mathcal{L}$ exceeding this number. (See Section 7.)

Proof of Theorem 2.1. By replacing $\mathcal{L}$ with a twist we may assume it has its first generator in degree $-(s-4)$. We then get

$$
0 \longrightarrow \mathcal{O}_{S}(s-4) \longrightarrow \mathcal{L} \longrightarrow \omega_{X} \longrightarrow 0,
$$

where the speciality index $e_{X} \leq s-4$. To prove the theorem it will be enough to establish the following three facts. 
1. $e_{X} \geq-2$.

2. The gap in the generators of $H_{*}^{0} \omega_{X}$ is $\leq s-2$.

3. If the last generator of $H_{*}^{0} \omega_{X}$ occurs in degree $d$, then the regularity of $H_{*}^{0} \omega_{X}$ is $\leq d+(s-2)$.

1. This obviously holds, since the speciality index of $X$ is greater or equal to that of any reduced, irreducible subcurve of $X$.

3. If the last generator of $H_{*}^{0} \omega_{X}$ occurs in degree $d$, then $\omega_{X}(d)$ is generated by its sections. Since $X$ is generically a Cartier divisor on $S, \omega_{X}(d)$ is generically an invertible sheaf on $X$. Then a general map $\mathcal{O}_{X} \rightarrow \omega_{X}(d)$ will have a cokernel $\mathcal{T}$ with finite support giving a sequence

$$
0 \longrightarrow \mathcal{O}_{X} \longrightarrow \omega_{X}(d) \longrightarrow \mathcal{T} \longrightarrow 0 \text {. }
$$

Since $e_{X} \leq s-4$, we get $H^{1} \mathcal{O}_{X}(s-3)=0$; so $H^{1} \omega_{X}(d+(s-3))=0$, implying that $\omega_{X}$ is $d+(s-2)$-regular.

2. Suppose there are minimal generators of $H_{*}^{0} \omega_{X}$ of degrees $d^{\prime}$ and $d^{\prime \prime}$ with $d^{\prime \prime}>d^{\prime}+(s-2)$, and that there is no minimal generator of $H_{*}^{0} \omega_{X}$ of degree between $d^{\prime}$ and $d^{\prime \prime}$. Let $\mathcal{M}$ be the subsheaf of $\omega_{X}$ generated by the generators of degree $\leq d^{\prime}$. The inclusion $\mathcal{M} \hookrightarrow \omega_{X}$ gives by Section 1 a sequence

$$
0 \longrightarrow \hat{\mathcal{M}} \longrightarrow \omega_{X} \longrightarrow \mathcal{A} \longrightarrow 0 \text {. }
$$

Applying the functor $\mathcal{H} m_{\mathcal{O}_{X}}\left(-, \omega_{X}\right)$, we get by Proposition 1.1 a sequence

$$
0 \longrightarrow \mathcal{H o m}_{\mathcal{O}_{X}}\left(\mathcal{A}, \omega_{X}\right) \longrightarrow \mathcal{O}_{X} \longrightarrow \mathcal{H}_{\text {om }_{\mathcal{O}_{X}}}\left(\hat{\mathcal{M}}, \omega_{X}\right) \longrightarrow 0
$$

Thus $\mathcal{H}_{\text {om }} \mathcal{O}_{X}\left(\hat{\mathcal{M}}, \omega_{X}\right)=\mathcal{O}_{Y}$ for some locally Cohen-Macaulay subcurve $Y$, and $\mathcal{H} m_{\mathcal{O}_{X}}\left(\mathcal{A}, \omega_{X}\right)=\mathcal{I}_{Y / X}$, an ideal subsheaf of $\mathcal{O}_{X}$. Dualizing back and using Proposition 1.1 , we see that the sequence (5) is then

$$
0 \longrightarrow \omega_{Y} \longrightarrow \omega_{X} \longrightarrow \mathcal{A} \longrightarrow 0 \text {. }
$$

Now since $\omega_{Y}\left(d^{\prime}\right)$ is generated by its sections except at a finite number of points, $\omega_{Y}$ is $d^{\prime}+(s-2)$ regular by the same argument as in 3 . Thus $Y \neq X$. We also see that the minimal generator of $H_{*}^{0} \omega_{Y}$ of highest degree is in degree $d^{\prime}$.

Next we claim that $H^{0} \mathcal{A}(n)=0$ for $n<d^{\prime \prime}$. This is because

$$
H^{1} \omega_{Y}\left(d^{\prime}+(s-3)\right)=0
$$

and the first degree of a minimal generator of $H_{*}^{0} \omega_{X}$ of degree $>d^{\prime}$ is $d^{\prime \prime}>d^{\prime}+$ $(s-2)$.

The idea is now to use Theorem 6.1 to show that this large gap between degrees of generators in $H_{*}^{0} \omega_{Y}$ and $H_{*}^{0} \mathcal{A}$ is impossible. Let $D$ be a reduced, irreducible curve in the support of $\mathcal{I}_{Y / X}$ (but the support of $\mathcal{I}_{Y / X}$ may be nonreduced along $D)$. Let $\overline{\mathcal{F}}=\left(\mathcal{I}_{Y / X}\right)_{\mid D^{\circ}}$. By pushdown we get a diagram

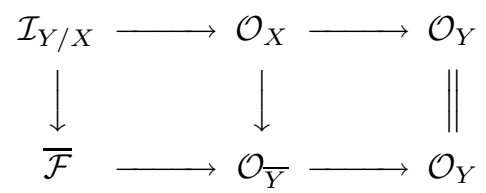

and a sequence

$$
0 \longrightarrow \mathcal{I}_{\bar{Y} / X} \longrightarrow \mathcal{I}_{Y / X} \longrightarrow \overline{\mathcal{F}} \longrightarrow 0
$$


Applying $\mathcal{H o m}_{\mathcal{O}_{X}}\left(-, \omega_{X}\right)$, we get a sequence

$$
0 \longrightarrow \mathcal{H o m}_{\mathcal{O}_{X}}\left(\overline{\mathcal{F}}, \omega_{X}\right) \longrightarrow \mathcal{A} \longrightarrow \mathcal{H o m}_{\mathcal{O}_{X}}\left(\mathcal{I}_{\bar{Y} / X}, \omega_{X}\right) \longrightarrow 0 .
$$

Let $\overline{\mathcal{G}}=\mathcal{H}^{\circ} m_{\mathcal{O}_{X}}\left(\overline{\mathcal{F}}, \omega_{X}\right)$. Then we see that $\overline{\mathcal{G}}$ has no sections in degrees $<d^{\prime \prime}$. Now distinguish two cases.

a. If $D$ is not a subcurve of $Y$, then $\overline{\mathcal{F}}$ is an ideal in $\mathcal{O}_{D}$. Thus

$$
\omega_{D}=\mathcal{H o m}_{\mathcal{O}_{D}}\left(\mathcal{O}_{D}, \omega_{X}\right) \subseteq \overline{\mathcal{G}} .
$$

But since $\omega_{D}(2)$ has sections (by Hilbert polynomial considerations), we see that $d^{\prime \prime} \leq 2$. This is impossible, since $d^{\prime} \geq-(s-4)$.

b. Suppose then that $D$ is a subcurve of $Y$. Let $Y^{\prime}$ be the residual of $D$ in $Y$, so we have a sequence

$$
0 \longrightarrow \mathcal{F} \longrightarrow \mathcal{O}_{Y} \longrightarrow \mathcal{O}_{Y^{\prime}} \longrightarrow 0
$$

Let $\mathcal{G}=\mathcal{H o m}_{\mathcal{O}_{Y}}\left(\mathcal{F}, \omega_{Y}\right)$, so we have a sequence

$$
0 \longrightarrow \omega_{Y^{\prime}} \longrightarrow \omega_{Y} \longrightarrow \mathcal{G} \longrightarrow 0 .
$$

Then, since $Y^{\prime} \neq Y$, clearly $\mathcal{G}\left(d^{\prime}\right)$ has a section. By Corollary 6.2 then also $\overline{\mathcal{G}}\left(d^{\prime}+(s-2)\right)$ has a section. This gives a contradiction.

\section{Curves on a CUBiC SURface}

In this section we wish to say something more about how the Hilbert function of the sheaf $\mathcal{L}$ behaves, where $\mathcal{L}$ is given as in Theorem 2.1. By replacing $\mathcal{L}$ with a twist so the minimal section is in degree $-(s-4)$ we get a sequence

$$
0 \longrightarrow \mathcal{O}_{S}(s-4) \longrightarrow \mathcal{L} \longrightarrow \omega_{X} \longrightarrow 0 .
$$

We study the Hilbert function of $\mathcal{L}$ by studying the Hilbert function of $\omega_{X}$. Let $p(n)=h^{0} \omega_{X}(n)$ and let $\Delta^{2} p$, be the second difference function.

In the following we restrict to the case where $S$ is a normal cubic surface. In this case we may derive a simple necessary condition on the behaviour of $\Delta^{2} p$. The methods are not in principle particular for a cubic surface, but formulating a precise theorem about the behaviour of $\Delta^{2} p$ when $s \geq 4$ seems to be more awkward, and proving it would be even more so.

We show the following (the proof is at the end of this section).

Theorem 3.1. Let $S$ be a normal cubic surface. Let $X$ be constructed as above. Then $\Delta^{2} p(n)$ is a non-negative weakly decreasing function for $n \geq 2$. For $n \gg 0$ we have $\Delta^{2} p(n)=0$.

Here $X$ is a curve with speciality index $e_{X} \leq s-4=-1$. Thus $X$ is minimal in its even biliaison class on the surface $S$. One might then ask what version might be true for a curve on $S$ not minimal in its even biliaison class on the surface $S$. It turns out that in this case it is more natural to formulate the theorem as a property of the Rao module of $X$.

Let

$$
\rho(n)=h^{1} \mathcal{I}_{X}(n)
$$


Then $\rho(n)=h^{0} \mathcal{O}_{X}(n)$ for $n<0$, and $\rho(0)=h^{0} \mathcal{O}_{X}-1$. From the two identities

$$
\begin{gathered}
h^{0} \mathcal{O}_{X}(n)-h^{1} \mathcal{O}_{X}(n)=d n+1-g, \\
h^{1} \mathcal{O}_{X}(n)=h^{0} \omega_{X}(-n),
\end{gathered}
$$

we deduce that for $n \leq-1$

$$
\begin{aligned}
\rho(n)-\rho(n-1) & =d+h^{0} \omega_{X}(-n)-h^{0} \omega_{X}(-n+1), \\
\Delta \rho(n) & =d-\Delta p(-n+1), \\
\Delta^{2} \rho(n) & =\Delta^{2} p(-n+2),
\end{aligned}
$$

and for $n=0$ we get

$$
\Delta^{2} \rho(n)=\Delta^{2} p(-n+2)-1 .
$$

Let $\delta_{a}$ be the Kronecker delta function, i.e. $\delta_{a}(n)=\delta_{n, a}$. Then for $n \leq 0$

$$
\Delta^{2} \rho(n)+\delta_{0}(n)=\Delta^{2} p(-n+2) .
$$

Theorem 3.2. Let $X$ be on a normal cubic surface $S$.

1. If $e_{X} \geq s-4=-1$, then $\Delta^{2} \rho(n)+\delta_{e_{X}-(s-4)}(n)$ is a weakly increasing function when $n \leq e_{X}-(s-4)$.

2. If $e_{X} \leq s-4=-1$, then $\Delta^{2} \rho(n)+\delta_{0}(n)$ is a weakly increasing function when $n \leq 0$.

Proof. We have the sequence

$$
0 \longrightarrow \mathcal{L} \longrightarrow \mathcal{O}_{S} \longrightarrow \mathcal{O}_{X} \longrightarrow 0
$$

and the Rao module $R_{X}=H_{*}^{1} \mathcal{L}$. Furthermore, $h^{1} \mathcal{O}_{X}\left(e_{X}\right) \neq 0$ gives $h^{2} \mathcal{L}\left(e_{X}\right) \neq 0$. Now

$$
\begin{aligned}
\left(H^{2} \mathcal{L}\left(e_{X}\right)\right)^{*} & =\operatorname{Ext}^{0}\left(\mathcal{L}\left(e_{X}\right), \omega_{S}\right) \\
& =\operatorname{Hom}\left(\mathcal{L}, \mathcal{O}_{S}\left(s-4-e_{X}\right)\right) .
\end{aligned}
$$

We thus get

$$
0 \longrightarrow \mathcal{L}\left(e_{X}-(s-4)\right) \longrightarrow \mathcal{O}_{S} \longrightarrow \mathcal{O}_{Y}
$$

where the speciality index $e_{Y} \leq s-4$, and the Rao module

$$
\begin{aligned}
R_{Y} & =H_{*}^{1} \mathcal{L}\left(e_{X}-(s-4)\right) \\
& =R_{X}\left(e_{X}-(s-4)\right) .
\end{aligned}
$$

The theorem now follows from Theorem 3.1 and (6)

By looking on the curve linked to $X$ we may also get a description of how the Rao module of $X$ behaves for larger values of $n$.

Theorem 3.3. Let $X$ be a curve on a normal cubic surface $S$. Let $t$ be minimal such that $X$ is on a complete intersection $(3, t)$. Then $\Delta^{2} \rho(n)+\delta_{t+1}(n)$ is weakly decreasing for $n \geq t+1$.

Proof. We have the sequence

$$
0 \longrightarrow \mathcal{L} \longrightarrow \mathcal{O}_{S} \longrightarrow \mathcal{O}_{X} \longrightarrow 0
$$

and the Rao module $R_{X}=H_{*}^{1} \mathcal{L}$. Also, the injection

$$
\mathcal{O}_{S}(-t) \longrightarrow \mathcal{L}
$$


gives a sequence

$$
0 \longrightarrow \mathcal{O}_{S}(s-4) \longrightarrow \mathcal{L}(t+s-4) \longrightarrow \omega_{D} \longrightarrow 0
$$

for a curve $D$. Here

$$
h^{1} \mathcal{L}(t+s-4+r)=h^{1} \omega_{D}(r)=h^{0} \mathcal{O}_{D}(-r)
$$

for $r \geq 1$, and

$$
h^{1} \mathcal{L}(t+s-4)=h^{0} \mathcal{O}_{D}-1
$$

Thus

$$
h^{1} \mathcal{L}(t+s-4+r)=h^{1} \mathcal{I}_{D}(-r)
$$

for $r \geq 0$.

Now we get for $r \geq 2$

$$
\begin{aligned}
\Delta^{2} \rho(t+s-4+r) & =\Delta^{2} h^{1} \mathcal{L}(t+s-4+r) \\
& =\Delta^{2} h^{1} \mathcal{I}_{D}(-r+2) .
\end{aligned}
$$

From this we see that $\Delta^{2} \rho+\delta_{t+1}$ is weakly decreasing for $n \geq 2+t+s-4=$ $t+1$.

Remark. From the sequence

$$
0 \longrightarrow \mathcal{L} \longrightarrow \mathcal{O}_{S} \longrightarrow \mathcal{O}_{X} \longrightarrow 0
$$

it follows that for $n \geq \max \left(s-3, e_{X}+1\right)$ we have $H^{2} \mathcal{L}(n)=0$. Furthermore, for $n<t, H^{0} \mathcal{L}(n)=0$. Thus

$$
\rho(n)=H^{1} \mathcal{L}(n)=-\chi \mathcal{L}(n)=\chi \mathcal{O}_{X}(n)-\chi \mathcal{O}_{S}(n)
$$

for

$$
\max \left(s-3, e_{X}+1\right) \leq n<t .
$$

This holds whether $S$ is normal or not (and regardless of the degree of $S$ ). The values of $\Delta^{2} \rho$ which we do not have any statement about are thus (assuming $s=3$ )

$$
\Delta^{2} \rho(t), \quad \Delta^{2} \rho\left(e_{X}+2\right) \quad\left(\text { resp. } \Delta^{2} \rho(1) \text { if } e_{X}=-2\right) \text {. }
$$

Curves with multiplicities on lines. In order to prove Theorem 3.1 we need to do some preliminary work. Let $L$ be a line on $S$ and let $R$ be another curve on $S$ with $R \cap L$ finite. The ideal $\mathcal{I}_{L}^{i} \cdot \mathcal{I}_{R}$ defines a subscheme of $S$ consisiting of a locally Cohen-Macaulay curve $X^{(i)}$ and some embedded points. As we shall see just after Corollary 6.4 , we get sequences

$$
0 \longrightarrow \mathcal{F}_{i-1} \longrightarrow \mathcal{O}_{X^{(i)}} \longrightarrow \mathcal{O}_{X^{(i-1)}} \longrightarrow 0,
$$

and if $L^{(i)}$ is the multiplicity $i$ structure on $L$ in $S$ we get sequences

$$
0 \longrightarrow \mathcal{G}_{i-1} \longrightarrow \mathcal{O}_{L^{(i)}} \longrightarrow \mathcal{O}_{L^{(i-1)}} \longrightarrow 0 \text {. }
$$

Lemma 3.4. Suppose $H^{0} \mathcal{O}_{X^{(i)}}(-1) \neq 0$ for some $i$. Then there are $m \geq 1$ and $v \geq 0$ such that

i. $\operatorname{deg} \mathcal{F}_{a}<0$ for $a<v$.

ii. $\mathcal{F}_{v}=\mathcal{F}_{v+1}=\cdots=\mathcal{F}_{v+m-1}=\mathcal{O}_{L}$.

iii. $\mathcal{F}_{a+m}=\mathcal{F}_{a}(1)$ for $a \geq v$. 
Proof. We see that $\mathcal{G}_{0}=\mathcal{O}_{L}$. From the sequence

$$
0 \longrightarrow \mathcal{G}_{1} \longrightarrow \mathcal{O}_{L^{(2)}} \longrightarrow \mathcal{O}_{L} \longrightarrow 0
$$

we see that $\mathcal{G}_{1}=\operatorname{Tor}_{1}^{\mathcal{O}_{S}}\left(\mathcal{O}_{L}, \mathcal{O}_{L}\right)^{\circ}$. The sequence (16) in Section 6 with $s=3$ and $\mathcal{F}=\mathcal{O}_{L}$ becomes

$$
0 \longrightarrow \mathcal{O}_{L}(-3) \longrightarrow \mathcal{O}_{L}(-1) \oplus \mathcal{O}_{L}(-1) \longrightarrow \operatorname{Tor}_{1}^{\mathcal{O}_{S}}\left(\mathcal{O}_{L}, \mathcal{O}_{L}\right) \longrightarrow 0
$$

so we get that $\operatorname{Tor}_{1}^{\mathcal{O}_{S}}\left(\mathcal{O}_{L}, \mathcal{O}_{L}\right)^{\circ}=\mathcal{O}_{L}(-1), \mathcal{O}_{L}$, or $\mathcal{O}_{L}(1)$.

By Theorem 6.1 we have

$$
\mathcal{G}_{i} \subseteq \mathcal{G}_{i-1}(1), \quad \mathcal{F}_{i} \subseteq \mathcal{F}_{i-1}(1) .
$$

Also, since $R \cap L$ is finite we have

$$
\mathcal{F}_{i} \subseteq \mathcal{G}_{i} .
$$

Now let $m$ be minimal such that $\operatorname{deg} \mathcal{G}_{m}>\operatorname{deg} \mathcal{G}_{m-1}$. (If no such $m$ exists, then clearly

$$
\operatorname{deg} \mathcal{F}_{i} \leq \operatorname{deg} \mathcal{G}_{i} \leq 0
$$

for all $i$, and $H^{0} \mathcal{O}_{X^{(i)}}(-1)=0$.) By (8) we get $\mathcal{G}_{m}=\mathcal{G}_{m-1}(1)$. From Theorem 6.5 we then get

$$
\mathcal{F}_{a+m}=\mathcal{F}_{a} \otimes \mathcal{G}_{m}
$$

for all $a \geq 0$.

a. If now $\mathcal{G}_{1}=\mathcal{O}_{L}(-1)$, we get $\operatorname{deg} \mathcal{G}_{m} \leq 0$ and

$$
\operatorname{deg} \mathcal{F}_{i} \leq \operatorname{deg} \mathcal{G}_{i} \leq 0
$$

for $i<m$. By (9) this gives $\operatorname{deg} \mathcal{F}_{a} \leq 0$ for all $a \geq 0$, and so $H^{0} \mathcal{O}_{X^{(i)}}(-1)=0$.

b. If $\mathcal{G}_{1}=\mathcal{O}_{L}(1)$, then $m=1$, and $\mathcal{F}_{a+1}=\mathcal{F}_{a}(1)$ for all $a \geq 0$.

c. So consider the case $\mathcal{G}_{1}=\mathcal{O}_{L}$. The non-zero map $\left(\mathcal{G}_{1}\right)^{\otimes i} \longrightarrow \mathcal{G}_{i}$ gives $\operatorname{deg} \mathcal{G}_{i} \geq 0$ for $i \geq 0$. Then $\mathcal{G}_{m}=\mathcal{O}_{L}(1)$ and

$$
\mathcal{G}_{0}=\mathcal{G}_{1}=\cdots=\mathcal{G}_{m-1}=\mathcal{O}_{L} .
$$

Let $v$ be minimal with $\operatorname{deg} \mathcal{F}_{v} \geq 0$. Then we must have $\mathcal{F}_{v}=\mathcal{O}_{L}$. We claim that

$$
\mathcal{F}_{v}=\mathcal{F}_{v+1}=\cdots=\mathcal{F}_{v+m-1}=\mathcal{O}_{L} .
$$

By (21) in Section 6 there are non-zero maps $\mathcal{F}_{i} \otimes \mathcal{G}_{1} \longrightarrow \mathcal{F}_{i+1}$. If $v \geq 1$, then $\mathcal{F}_{v-1}=\mathcal{O}_{L}(-1)$ and

$$
\mathcal{F}_{v+m-1}=\mathcal{F}_{v-1}(1)=\mathcal{O}_{L} .
$$

If $v=0$, then

$$
\operatorname{deg} \mathcal{F}_{v+m-1}=\operatorname{deg} \mathcal{F}_{m-1} \leq \operatorname{deg} \mathcal{G}_{m-1} \leq 0 .
$$

We now show the following, which is equivalent to Theorem 3.1 by (6).

Theorem 3.5. The function $\Delta^{2} \rho(n)+\delta_{0}(n)$ of $X$ is weakly increasing in the range $n \leq 0$. 
Proof. Let $-r$ be minimal such that $H^{0} \mathcal{O}_{X}(-r) \neq 0$. Let $\mathcal{F}$ be the subsheaf of $\mathcal{O}_{X}(-r)$ generated by its sections $H^{0} \mathcal{O}_{X}(-r)$. By Section 1 we have a sequence

$$
0 \longrightarrow \hat{\mathcal{F}} \longrightarrow \mathcal{O}_{X} \longrightarrow \mathcal{O}_{Y} \longrightarrow 0,
$$

where $Y$ is a locally Cohen-Macaulay subcurve of $X$. By Corollary 6.4 the support of $\hat{\mathcal{F}}$ is set-theoretically the disjoint union of lines, say $L_{1}, L_{2}, \ldots, L_{h}$. Let $L_{i}$ occur with multiplicity $n_{i}$ in $X$, so $X=L_{i}^{\left(n_{i}\right)} \cup R_{i}$ for some curve $R_{i}$ with finite intersection with $L_{i}$. We then get a filtration as in (7):

$$
0 \longrightarrow \mathcal{F}_{i, j-1} \longrightarrow \mathcal{O}_{L_{i}^{(j)} \cup R_{i}} \longrightarrow \mathcal{O}_{L_{i}^{(j-1)} \cup R} \longrightarrow 0 .
$$

By Lemma 3.4 there are $m_{i} \geq 1$ and $v_{i} \geq 0$ such that

$$
\mathcal{F}_{i, v_{i}}=\mathcal{F}_{i, v_{i}+1}=\cdots=\mathcal{F}_{i, v_{i}+m_{i}-1}=\mathcal{O}_{L_{i}}
$$

and

$$
\mathcal{F}_{i, a+m_{i}}=\mathcal{F}_{i, a}(1)
$$

for $a \geq v_{i}$. Write $n_{i}=v_{i}+t_{i}+r_{i} m_{i}$, where $0 \leq t_{i}<m_{i}$. Note that we must have $\mathcal{F}_{i, n_{i}}=\mathcal{O}_{L_{i}}(r)$.

Now let

$$
X^{\prime}=L_{1}^{\left(v_{1}+r_{1} m_{1}-1\right)} \cup \cdots \cup L_{h}^{\left(v_{h}+r_{h} m_{h}-1\right)} \cup R .
$$

Then there is a sequence

$$
0 \longrightarrow \mathcal{K} \longrightarrow \mathcal{O}_{X} \longrightarrow \mathcal{O}_{X^{\prime}} \longrightarrow 0
$$

where $\mathcal{K}$ is the kernel.

We now see the following:

1. $h^{0} \mathcal{O}_{X^{\prime}}(-r-1)=0$.

2. $h^{0} \mathcal{K}(-r)=\sum_{i=1}^{h}\left(t_{i}+1\right)$.

3. $h^{0} \mathcal{K}(-r+j)=(j+1) \sum_{i=1}^{h}\left(t_{i}+1\right)$ for $j \geq 0$.

4. $h^{0} \mathcal{O}_{X^{\prime}}(-r)=0$. This is because a section of $\mathcal{O}_{X^{\prime}}(-r)$ must lift to a section of $\mathcal{O}_{X}(-r)$. It would therefore have to be set-theoretically supported on a subset of the lines $L_{i}$. But by Lemma 3.4 applied to $X^{\prime}$ this is impossible.

5. $h^{0} \mathcal{O}_{X^{\prime}}(-r+1) \geq \sum_{i=1}^{h} m_{i} \geq \sum_{i=1}^{h}\left(t_{i}+1\right)$. This follows again by Lemma 3.4.

From this we now see the following:

a. $\Delta^{2} \rho_{X}(n)=\Delta^{2} \rho_{X^{\prime}}(n)$ for $n \in[-r+1,0]$.

b. $\Delta^{2} \rho_{X}(-r+1)=\Delta^{2} \rho_{X^{\prime}}(-r+1) \geq \sum_{i=1}^{h}\left(t_{i}+1\right)$.

c. $\Delta^{2} \rho_{X}(-r)=\sum_{i=1}^{h}\left(t_{i}+1\right)$.

By induction on $\operatorname{deg} X$ we then see that $\Delta^{2} \rho_{X}(n)+\delta_{0}(n)$ is weakly increasing for $n \leq 0$.

\section{INTERPRETATION BY INITIAL IDEALS}

In this section we wish to give interpretations of the results in the two preceding sections via initial ideals. The reason is that the initial ideal and the higher initial ideal are simple natural objects associated to a space curve, and they give a fairly refined invariant of the space curve which is nicely represented in a plane diagram and contains a lot of information. For instance, from the initial ideal and the higher initial ideal one may read all the cohomological dimensions $h^{i} \mathcal{I}_{X}(n)$ of a curve $X$. 
We therefore see it as a goal to understand which initial ideals and higher initial ideals can be associated to a space curve. Actually it is by considerations about the initial ideal that we were led to the theorems in Sections 1 and 2.

Now $X \subseteq S$ is a curve on a normal surface $S \subseteq \mathbf{P}^{3}$ of degree $s$. Let $I \subseteq$ $k\left[x_{0}, x_{1}, x_{2}, x_{3}\right]$ be its homogeneous ideal and in $(I)$ its initial ideal for the reverse lexicographic order. (See Ei], 15.2.)

Theorem 4.1. Let

$$
\operatorname{in}(I)=\left(\mathbf{x}^{I_{0}}, \ldots, \mathbf{x}^{I_{t}}\right)
$$

be a minimal generator set. Consider the set

$$
\left\{\mathbf{x}^{I_{0}}, \ldots, \mathbf{x}^{I_{t}}\right\} \backslash\left\{x_{0}^{s}\right\} .
$$

Let the elements have degree

$$
n_{0} \leq n_{1} \leq \cdots \leq n_{t^{\prime}} .
$$

Then $n_{j}-n_{j-1} \leq s-2$ for $j=1, \ldots, t^{\prime}$.

In other words, the gap between the degrees of the minimal generators in in $(I)$, excluding $x_{0}^{s}$ if that is a minimal generator, is $\leq s-2$.

Proof. There is a sequence

$$
0 \longrightarrow \mathcal{O}_{\mathbf{P}^{3}}(-s) \longrightarrow \mathcal{I}_{X} \longrightarrow \mathcal{L} \longrightarrow 0,
$$

where $\mathcal{L}$ is the ideal of $X$ in $S$. Let the minimal generators of $H_{*}^{0} \mathcal{L}$ be in degrees

$$
m_{0} \leq m_{1} \leq \cdots \leq m_{r}
$$

If $m_{r}=1$, then $X$ is a line and the theorem holds. So assume $m_{r} \geq 2$. The ideal $I$ will also have minimal generators in these degrees and thus also in $(I)$ (being a degeneration of $I$ ). Now the regularity

$$
\operatorname{reg} \operatorname{in}(I)=\operatorname{reg} I \leq \max (s, \operatorname{reg} \mathcal{L}) \leq m_{r}+(s-2) .
$$

Then the biggest generator of in $(I)$ occurs in degree $\leq m_{r}+(s-2)$ by [Ba-St]. Furthermore, the only possible generator of in $(I)$ in degree $<m_{0}$ will be $x_{0}^{s}$. Hence the theorem follows.

Again we must say that the theorem does not hold when the suface $S$ is not normal. Then the gaps between the degrees of the generators of in $(I)$ may be arbitrarily large.

When giving the analogous statement for the higher initial ideal it is most natural to do so for curves that are minimal in their biliaison class on the surface $S$. So let $X$ be such a curve, i.e. a curve with speciality index $e_{X} \leq s-4$.

The higher initial ideal $\operatorname{in}_{1}(I)$ of $X$ consists of $T^{2} \subseteq R^{2}$. (See [Fl] or [Fl-Gr].)

Theorem 4.2. Let the minimal generators of $T^{2}$ occur in degrees

$$
n_{0} \leq n_{1} \leq \cdots \leq n_{t} .
$$

Then $n_{j}-n_{j-1} \leq s-2$ for $j=1, \ldots, t$.

Proof. There is a sequence

$$
0 \longrightarrow \mathcal{O}_{S}(s-4) \longrightarrow \mathcal{L} \longrightarrow \omega_{X} \longrightarrow 0,
$$

where $H_{*}^{0} \mathcal{L}$ has its minimal generator in degree $-(s-4)$. Let the other generators 
of $H_{*}^{0} \mathcal{L}$ occur in degrees

$$
m_{0} \leq m_{1} \leq \cdots \leq m_{u}
$$

These will also be the degrees of the minimal generators of $H_{*}^{0} \omega_{X}$.

Now the resolution of $T^{2}$ is a specialisation of the resolution of $\omega_{X}(4)$. (See [Fl], Section 4.) Thus $T^{2}(-4)$ also has generators in these degrees. To conclude we must show that the degree of the largest degree generator of $T^{2}(-4)$ has degree $\leq m_{u}+(s-2)$.

We show that the regularity of $\omega_{X}$ is greater or equal to the degree of the highest degree generator of $T^{2}(-4)$. Since the regularity of $\mathcal{L}$ is $\leq m_{u}+(s-2)$ with $m_{u} \geq-(s-4)$, we get that the regularity of $\omega_{X}$ is $\leq m_{u}+(s-2)$, and the theorem follows.

So let $r$ be the regularity of $\omega_{X}$. Then

$$
H^{1} \omega_{X}(r-2) \neq 0, \quad H^{1} \omega_{X}(r-1)=0
$$

so

$$
H^{0} \mathcal{O}_{X}(-r+2) \neq 0, \quad H^{0} \mathcal{O}_{X}(-r+1)=0 .
$$

This gives $r \geq 2$. Let the generator of $T^{2}(-4)$ of highest degree occur in degree $m$. Suppose $m \geq 3$. There is a sequence ([Fl], 11.4)

$$
0 \longrightarrow T^{2} \longrightarrow R^{2} \longrightarrow P_{1}^{2} \longrightarrow 0 \text {. }
$$

By [Fl], Section 11, there is a sequence

$$
0 \longrightarrow T_{x_{3}}^{2} \longrightarrow R_{x_{3}}^{2} \longrightarrow P_{1-}^{2} \longrightarrow 0
$$

such that (10) is just (11) tensored with $\bigotimes_{k} k\left[x_{3}\right]$. Now $R_{x_{3}}^{2}$ is generated by elements of degree $\leq-2$. The submodule $T_{x_{3}}^{2}$ has a generator in degree $m-4 \geq-1$. But then $P_{1-}^{2}$ must have an element in degree $m-5$. This gives $H^{1} \mathcal{I}_{X}(2-m) \neq 0$ ([F], 12.10), which implies $H^{0} \mathcal{O}_{X}(2-m) \neq 0$. Thus $-r+1<2-m$, giving $r \geq m$.

Examples 1 and 2 of the introduction are applications of Theorem 4.1.

Example 4. Suppose $S$ is a normal quadric surface (i.e. $S$ is reduced and irreducible). Let $X$ be a curve on $S$ minimal in its even liaison class on $S$. By Theorem $4.2, \operatorname{in}_{1}\left(I_{X}\right)$ must have the following form.

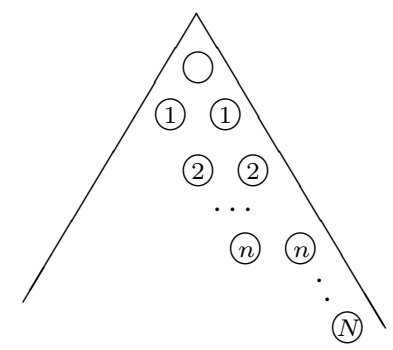


By [Fl-Gr], if $C$ is any curve on $S$ with $X$ as a minimal curve in its even liaison class on $S$, then $\operatorname{in}_{1}\left(I_{C}\right)$ must look like the following.

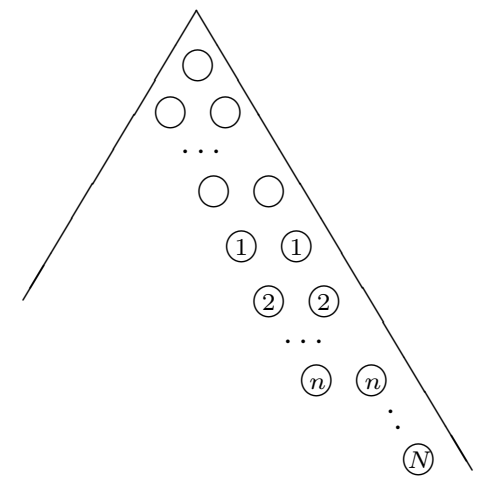

Analogously to Example 1 in the introduction, it follows that one must have $N=n$ or $n+1$.

\section{INITIAL IDEAL OF CURVES ON NORMAL CUBIC SURFACES}

We now wish to give the initial ideal version of the theorems of Section 3. We suppose that $X \subseteq S \subseteq \mathbf{P}^{3}$, where $S$ is a normal cubic surface. Let $I$ be the homogeneous ideal of $X$. Then we get the initial ideal in $(I)$ and the higher initial ideal $\operatorname{in}_{1}(I)$ represented by $T^{2} \subseteq R^{2}$.

Let $t$ be minimal such that $X$ is on a complete intersection $(3, t)$. That is, $t$ is minimal such that $x_{0}^{2} x_{1}^{t-2} \in \operatorname{in}(I)$.

Theorem 5.1. Let $l(\nu)$ be the number of minimal generators of in $I_{\nu}$ divisible by $x_{2}$. Then $l(\nu)+\delta_{t+1}(\nu)$ is weakly decreasing for $\nu \geq t+1$.

Let $e$ be the speciality index of $X$; that is, $e$ is maximal such that $\left(x_{1}^{e+2}\right)^{*}(2) \in$ $T^{2}$. (Exception: if the speciality index of $X$ is -2 , let $e=-1$.)

Theorem 5.2. Let $m(\nu)$ be the number of minimal generators of $T_{\nu}^{2}$ of the form $\left(x_{0}^{a} x_{1}^{b}\right)^{*}(2) \cdot x_{2}^{c}$, where $c \geq 1$. Then $m(\nu)+\delta_{-e-3}(\nu)$ is weakly decreasing for $\nu \geq$ $-e-3$.

We first give some notation. Let $I_{x_{3}}$ be the image of the map

$$
I \longrightarrow k\left[x_{0}, x_{1}, x_{2}, x_{3}\right] \longrightarrow k\left[x_{0}, x_{1}, x_{2}\right] .
$$

Then $\operatorname{in}\left(I_{x_{3}}\right)=\operatorname{in}(I)_{x_{3}}$, since the order is revlex. Let

$$
L_{0}=\operatorname{in}(I)_{x_{3}} \text { sat } / \operatorname{in}(I)_{x_{3}}
$$

and let $L_{0, \nu}$ be the graded piece of degree $\nu$. Also put

$$
l_{0}(\nu)=\operatorname{dim}_{k} L_{0, \nu} .
$$

Recalling that $R_{X}$ is the Rao module, from [F], $12.12 \mathrm{c}$ ), it follows that

$$
l_{0}(\nu)=\operatorname{dim}_{k} \operatorname{ker}\left(R_{X, \nu-1} \stackrel{\cdot h}{\longrightarrow} R_{X, \nu}\right)
$$

where $h$ is a general linear form. 
Now for the proof of Theorem 5.1.

Proof. Note that if $C$ is a complete intersection then 4.1 holds trivially. So assume $C$ is not a complete intersection. First we show that $x_{0}^{2} x_{1}^{t-1}, x_{0} x_{1}^{t}$ and $x_{1}^{t+1}$ are all in in $(I)$. Suppose the diagram for in $(I)$ is

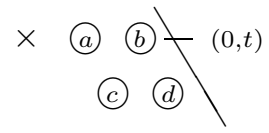

Then, since $C$ is contained in a complete intersection $(3, t)$, we must have $c=0$, and since $C$ is not a complete intersection, $d$ must be a finite number. By linking $(3, t)$ we get by [Fl-Gr] a curve $D$ where $\operatorname{in}_{1}\left(I_{D}\right)$ has diagram

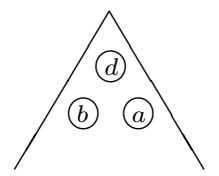

By Borel-fixedness of $i_{1}\left(I_{D}\right)$ one sees readily that if $d>0$ then $T_{-2}^{2}=0$, giving $h^{2} \mathcal{I}_{D}(-2)=0$. But any curve has speciality index $\geq-2$, so this is impossible. Thus $d=0$, and the above mentioned monomials are all in in $(I)$.

Let $\Gamma=X \cap H$ be a general hyperplane section and $J=I_{\Gamma}$. Since $\operatorname{in}(J)=$ $\operatorname{in}(I)_{x_{3}}{ }^{\text {sat }}$ we see that in $(J)$ is generated by monomials of degree $\leq t+1$, and hence $J$ is $(t+1)$-regular, [Ba-St], giving $H^{1} \mathcal{I}_{\Gamma}(\nu)=0$ for $\nu \geq t$.

The sequence

$$
0 \longrightarrow \mathcal{I}_{X}(-1) \stackrel{\cdot h}{\longrightarrow} \mathcal{I}_{X} \longrightarrow \mathcal{I}_{\Gamma} \longrightarrow 0
$$

gives

$$
H^{1} \mathcal{I}_{X}(\nu-1) \stackrel{\cdot h}{\longrightarrow} H^{1} \mathcal{I}_{X}(\nu) \longrightarrow H^{1} \mathcal{I}_{\Gamma}(\nu),
$$

implying that

$$
R_{X, \nu-1} \longrightarrow R_{X, \nu}
$$

is surjective for $\nu \geq t$. Thus $l_{0}(\nu)=-\Delta \rho(\nu)$ for $\nu \geq t$.

Consider the map

$$
L_{0, \nu-1} \stackrel{\cdot x_{2}}{\longrightarrow} L_{0, \nu} .
$$

We claim it is surjective for $\nu \geq t+1$. Assume to the contrary that there is an $m \in \operatorname{in}(I)_{x_{3}}{ }^{\text {sat }}$ such that $\bar{m}$ is not in the image. Let $m=x_{0}^{a} x_{1}^{b} x_{2}^{c}$. Then $x_{0}^{a} x_{1}^{b}$ must be in the saturation in $(I)_{x_{3}}{ }^{\text {sat }}$. If $c>0$ this implies that $\bar{m}$ is in the image.

If $c=0$, then $m \notin \operatorname{in}(I)_{x_{3}}$ but $m \in \operatorname{in}(I)_{x_{3}}{ }^{\text {sat }}=\operatorname{in}(J)$. From the first thing shown in this proof this implies $\operatorname{deg} m \leq t$. Thus the map is surjective for $\nu \geq t+1$. 
The kernel has a basis consisting of monomials $\bar{m}$ such that $m x_{2}$ is a minimal generator of in $(I)$. All such generators of in $(I)$ of degree $\nu \geq t+1$ occur this way.

Thus

$$
\Delta^{2} \rho(\nu)=-\Delta l_{0}(\nu)=l(\nu),
$$

the number of minimal generators of in $(I)_{\nu}$ divisible by $x_{2}$.

Remark. Example 3 of the introduction is an application of Theorem 5.1.

The proof of Theorem 5.2 is quite analogous to the above proof. We give a sketch. The higher initial ideal of $I$ is represented by $T^{2} \subseteq R^{2}$. We get the module $P_{1-}^{2}$ from the sequence (10) in Section 4, and let

$$
p_{1-}^{2}(\nu)=\operatorname{dim}_{k} P_{1-, \nu}^{2} .
$$

Then, by [F] , 12.10,

$$
p_{1-}^{2}(\nu)=\operatorname{dim}_{k} \operatorname{coker}\left(R_{X,-\nu-4} \stackrel{\cdot h}{\longrightarrow} R_{X,-\nu-3}\right) .
$$

Now first show that

$$
\left(x_{0}^{2} x_{1}^{e-1}\right)^{*}(2),\left(x_{0} x_{1}^{e}\right)^{*}(2),\left(x_{1}^{e+1}\right)^{*}(2)
$$

are all in $T^{2}$

Then show that

$$
R_{X, \mu-1} \stackrel{\cdot h}{\longrightarrow} R_{X, \mu}
$$

is injective for $\mu \leq e-1$.

Finally, show that

$$
P_{1-, \nu-1}^{2} \stackrel{\cdot x_{2}}{\longrightarrow} P_{1-, \nu}^{2}
$$

is surjective for $\nu \geq-e-3$ and that the kernel is generated by elements

$$
\overline{\left(x_{0}^{a} x_{1}^{b}\right)^{*}(2) \cdot x_{2}^{c-1}},
$$

where $c \geq 1$, such that $\left(x_{0}^{a} x_{1}^{b}\right)^{*}(2) \cdot x_{2}^{c}$ is a minimal generator of $T^{2}$.

\section{Technical RESUlts}

In this section we develop the necessary theory about non-reduced curves on normal surfaces in $\mathbf{P}^{3}$ required to yield the results in the previous sections.

The difference between normal surfaces and non-normal surfaces for the matters studied here is that if $S \subseteq \mathbf{P}^{3}$ is a surface and $D \subseteq S$ is a reduced and irreducible curve on $S$, then a multiplicity $n$ structure on $D$ is uniquely determined if $S$ is normal, but if $S$ is non-normal then there may be many (in fact infinitely many) such structures.

Suppose from now on that $S$ is normal. Let $\bar{X}$ be a curve on $S$ and let $D$ be any locally Cohen-Macaulay subcurve of $\bar{X}$. Let $X$ be the residual of $D$ in $\bar{X}$. Suppose that $D$ is also a subscheme of $X$, and let $X^{\prime}$ be the residual of $D$ in $X$. We then get two exact sequences:

$$
\begin{aligned}
0 \longrightarrow \mathcal{F} \longrightarrow \mathcal{O}_{X} \longrightarrow \mathcal{O}_{X^{\prime}} \longrightarrow 0 \\
0 \longrightarrow \overline{\mathcal{F}} \longrightarrow \mathcal{O}_{\bar{X}} \longrightarrow \mathcal{O}_{X} \longrightarrow 0
\end{aligned}
$$

where $\mathcal{F}$ and $\overline{\mathcal{F}}$ are the kernels. By Section 1 they are generically invertible sheaves on $D$. 
Let $\mathcal{I}_{X / S}$ be the ideal of $X$ in $S$ and $\mathcal{I}_{X^{\prime} / S}$ the ideal of $X^{\prime}$ in $S$. From the diagram

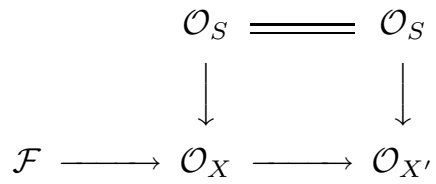

one gets the sequence

$$
0 \longrightarrow \mathcal{I}_{X / S} \longrightarrow \mathcal{I}_{X^{\prime} / S} \longrightarrow \mathcal{F} \longrightarrow 0 .
$$

Since $X^{\prime}$ is generically a Cartier divisor on $S$, this gives that

$$
\left(\mathcal{I}_{X^{\prime} / S} \otimes \mathcal{O}_{D}\right)^{\circ}=\mathcal{F} \text {, }
$$

where ${ }^{\circ}$ denotes the torsion free part. Correspondingly,

$$
\left(\mathcal{I}_{X / S} \otimes \mathcal{O}_{D}\right)^{\circ}=\overline{\mathcal{F}}
$$

The following says something about the relation between $\mathcal{F}$ and $\overline{\mathcal{F}}$.

Theorem 6.1. $\quad$ 1. $\mathcal{I}_{X / S} \otimes \mathcal{O}_{D}$, $\operatorname{Tor}_{1}^{\mathcal{O}_{S}}\left(\mathcal{F}, \mathcal{O}_{D}\right)$ and $\mathcal{H o m}_{\mathcal{O}_{D}}\left(\omega_{D}, \mathcal{F}\right)(s-4)$ are all generically invertible sheaves on $D$ whose classes in the Grothendieck group are equal.

2. There are generical isomorphisms

$$
\operatorname{Tor}_{1}^{\mathcal{O}_{S}}\left(\mathcal{F}, \mathcal{O}_{D}\right) \longrightarrow \mathcal{I}_{X / S} \otimes \mathcal{O}_{D} \longrightarrow \mathcal{H o m}_{\mathcal{O}_{D}}\left(\omega_{D}, \mathcal{F}\right)(s-4)
$$

In particular there is an injection

$$
\overline{\mathcal{F}} \hookrightarrow \mathcal{H o m}_{\mathcal{O}_{D}}\left(\omega_{D}, \mathcal{F}\right)(s-4) .
$$

Proof. 1. Let $\mathcal{I}_{X}$ be the ideal sheaf of $X$ in $\mathbf{P}^{3}$ and $\mathcal{I}_{X^{\prime}}$ the ideal sheaf of $X^{\prime}$ in $\mathbf{P}^{3}$. By tensoring

$$
0 \longrightarrow \mathcal{I}_{X} \longrightarrow \mathcal{O}_{\mathbf{P}^{3}} \longrightarrow \mathcal{O}_{X} \longrightarrow 0
$$

with $\mathcal{O}_{D}$ we get in the Grothendieck group of sheaves on $\mathbf{P}^{3}$ that

$$
\begin{aligned}
{\left[\mathcal{O}_{D}\right] } & =\sum_{i \geq 0}(-1)^{i}\left[\operatorname{Tor}_{i}^{\mathcal{O}_{\mathbf{P}^{3}}}\left(\mathcal{O}_{\mathbf{P}^{3}}, \mathcal{O}_{D}\right)\right] \\
& =\sum_{i \geq 0}(-1)^{i}\left[\operatorname{Tor}_{i}^{\mathcal{O}_{\mathbf{P}} 3}\left(\mathcal{I}_{X}, \mathcal{O}_{D}\right)\right]+\sum_{i \geq 0}(-1)^{i}\left[\operatorname{Tor}_{i}^{\mathcal{O}_{\mathbf{P}^{3}}}\left(\mathcal{O}_{X}, \mathcal{O}_{D}\right)\right] .
\end{aligned}
$$

Since $\mathcal{O}_{D}$ has local projective dimension 2 , there is a resolution of locally free sheaves on $\mathbf{P}^{3}$.

$$
0 \longrightarrow \mathcal{E}_{2} \longrightarrow \mathcal{E}_{1} \longrightarrow \mathcal{E}_{0} \longrightarrow \mathcal{O}_{D} \longrightarrow 0
$$

Thus

$$
\sum_{i \geq 0}(-1)^{i}\left[\operatorname{Tor}_{i}^{\mathcal{O}_{\mathbf{P}^{3}}}\left(\mathcal{O}_{X}, \mathcal{O}_{D}\right)\right]=0
$$

Since furthermore

$$
\operatorname{Tor}_{i}^{\mathcal{O}_{\mathbf{P}^{3}}}\left(\mathcal{I}_{X}, \mathcal{O}_{D}\right)=\operatorname{Tor}_{i+1}^{\mathcal{O}_{\mathbf{P} 3}}\left(\mathcal{O}_{X}, \mathcal{O}_{D}\right)
$$


for $i \geq 1$, we get

$$
\operatorname{Tor}_{i}^{\mathcal{O}_{\mathbf{P}^{3}}}\left(\mathcal{I}_{X}, \mathcal{O}_{D}\right)=0
$$

for $i \geq 2$ and $\operatorname{Tor}_{1}^{\mathcal{O}_{\mathrm{P}^{3}}}\left(\mathcal{I}_{X}, \mathcal{O}_{D}\right)$ is torsion free of rank one on $D$.

Tensoring the sequence

$$
0 \longrightarrow \mathcal{I}_{X} \longrightarrow \mathcal{I}_{X^{\prime}} \longrightarrow \mathcal{F} \longrightarrow 0
$$

with $\mathcal{O}_{D}$ gives a sequence

$$
0 \longrightarrow \operatorname{Tor}_{2}^{\mathcal{O}_{\mathbf{P}^{3}}}\left(\mathcal{F}, \mathcal{O}_{D}\right) \longrightarrow \operatorname{Tor}_{1}^{\mathcal{O}_{\mathbf{P}^{3}}}\left(\mathcal{I}_{X}, \mathcal{O}_{D}\right) \longrightarrow \operatorname{Tor}_{1}^{\mathcal{O}_{\mathbf{P}^{3}}}\left(\mathcal{I}_{X^{\prime}}, \mathcal{O}_{D}\right)
$$

Since all these sheaves are torsion free of rank one, the first map must be an isomorphism.

Hence we get from (14) that

$$
\left[\operatorname{Tor}_{2}^{\mathcal{O}^{3}}\left(\mathcal{F}, \mathcal{O}_{D}\right)\right]=\left[\operatorname{Tor}_{1}^{\mathcal{O}_{P^{3}}}\left(\mathcal{I}_{X}, \mathcal{O}_{D}\right)\right]=\left[\mathcal{I}_{X} \otimes \mathcal{O}_{D}\right]-\left[\mathcal{O}_{D}\right] .
$$

From the sequence

$$
0 \longrightarrow \mathcal{O}_{\mathbf{P}^{3}}(-s) \longrightarrow \mathcal{I}_{X} \longrightarrow \mathcal{I}_{X / S} \longrightarrow 0
$$

we get

$$
0 \longrightarrow \mathcal{O}_{D}(-s) \longrightarrow \mathcal{I}_{X} \otimes \mathcal{O}_{D} \longrightarrow \mathcal{I}_{X / S} \otimes \mathcal{O}_{D} \longrightarrow 0,
$$

giving

$$
\left[\mathcal{I}_{X} \otimes \mathcal{O}_{D}\right]=\left[\mathcal{I}_{X / S} \otimes \mathcal{O}_{D}\right]+\left[\mathcal{O}_{D}(-s)\right]
$$

so

$$
\begin{aligned}
{\left[\mathcal{I}_{X / S} \otimes \mathcal{O}_{D}\right] } & =\left[\mathcal{I}_{X} \otimes \mathcal{O}_{D}\right]-\left[\mathcal{O}_{D}(-s)\right] \\
& =\left[\operatorname{Tor}_{2}^{\mathcal{O}_{P^{3}}}\left(\mathcal{F}, \mathcal{O}_{D}\right)(s)\right] .
\end{aligned}
$$

We now wish to show that

$$
\begin{gathered}
\operatorname{Tor}_{2}^{\mathcal{O}_{\mathbf{P}^{3}}}\left(\mathcal{F}, \mathcal{O}_{D}\right)(s)=\mathcal{H o m}_{\mathcal{O}_{D}}\left(\omega_{D}, \mathcal{F}(s-4)\right), \\
{\left[\operatorname{Tor}_{2}^{\mathcal{O}_{\mathbf{P}^{3}}}\left(\mathcal{F}, \mathcal{O}_{D}\right)(s)\right]=\left[\operatorname{Tor}_{1}^{\mathcal{O}_{S}}\left(\mathcal{F}, \mathcal{O}_{D}\right)\right] .}
\end{gathered}
$$

To show 1a) we apply the functor $\mathcal{H o m}_{\mathcal{O}_{\mathbf{P}}^{3}}\left(-, \omega_{\mathbf{P}^{3}}\right)$ to the complex (15) and get a resolution

$$
0 \longrightarrow \mathcal{E}_{0}^{\vee} \longrightarrow \mathcal{E}_{1}^{\vee} \longrightarrow \mathcal{E}_{2}^{\vee} \longrightarrow \omega_{D}(4) \longrightarrow 0
$$

Applying $\mathcal{H o m}_{\mathcal{O}_{D}}(-, \mathcal{F})$ to this, we get

$$
0 \longrightarrow \mathcal{H o m}_{\mathcal{O}_{D}}\left(\omega_{D}, \mathcal{F}(-4)\right) \longrightarrow \mathcal{F} \otimes \mathcal{E}_{2} \longrightarrow \mathcal{F} \otimes \mathcal{E}_{1},
$$

so we see that

$$
\operatorname{Tor}_{2}^{\mathcal{O}_{\mathbf{P}^{3}}}\left(\mathcal{F}, \mathcal{O}_{D}\right)=\mathcal{H o m}_{\mathcal{O}_{D}}\left(\omega_{D}, \mathcal{F}(-4)\right)
$$

Now we prove 1b). From [Ei], Exercise A3.45, there exist to an algebra morphism $R \rightarrow S$, an $R$-module $B$ and an $S$-module $A$ associated a spectral sequence

$$
{ }^{2} E^{p q}=\operatorname{Tor}_{q}^{S}\left(\operatorname{Tor}_{p}^{R}(S, B), A\right) \Rightarrow_{p} \operatorname{Tor}_{p+q}^{R}(B, A) .
$$

Applying this with

$$
S=\mathcal{O}_{S}, \quad R=\mathcal{O}_{\mathbf{P}^{3}}, \quad A=\mathcal{O}_{D}, \quad B=\mathcal{F},
$$


we get a sequence

$$
0 \longrightarrow \mathcal{F}(-s) \longrightarrow \operatorname{Tor}_{1}^{\mathcal{O}^{3}}\left(\mathcal{F}, \mathcal{O}_{D}\right) \longrightarrow \operatorname{Tor}_{1}^{\mathcal{O}_{S}}\left(\mathcal{F}, \mathcal{O}_{D}\right) \longrightarrow 0
$$

Again from (15) we see that

$$
\left[\operatorname{Tor}_{1}^{\mathcal{O}_{\mathbf{P}^{3}}}\left(\mathcal{F}, \mathcal{O}_{D}\right)\right]=\left[\operatorname{Tor}_{2}^{\mathcal{O}_{\mathbf{P}} 3}\left(\mathcal{F}, \mathcal{O}_{D}\right)\right]+[\mathcal{F}] .
$$

Hence (16) gives that

$$
\begin{aligned}
& {\left[\operatorname{Tor}_{1}^{\mathcal{O}_{S}}\left(\mathcal{F}, \mathcal{O}_{D}\right)\right]=\left[\operatorname{Tor}_{2}^{\mathcal{O}^{3}}\left(\mathcal{F}, \mathcal{O}_{D}\right)\right]+[\mathcal{F}]-[\mathcal{F}(-s)]} \\
& =\left[\operatorname{Tor}_{2}^{\mathcal{O}_{\mathbf{P}}^{3}}\left(\mathcal{F}, \mathcal{O}_{D}\right)(s)\right]
\end{aligned}
$$

2. The sequence

$$
0 \longrightarrow \mathcal{I}_{X / S} \longrightarrow \mathcal{I}_{X^{\prime} / S} \longrightarrow \mathcal{F} \longrightarrow 0
$$

gives

$$
\operatorname{Tor}_{1}^{\mathcal{O}_{S}}\left(\mathcal{F}, \mathcal{O}_{D}\right) \longrightarrow \mathcal{I}_{X / S} \otimes \mathcal{O}_{D} \longrightarrow \mathcal{I}_{X^{\prime} / S} \otimes \mathcal{O}_{D} \longrightarrow \mathcal{F} \longrightarrow 0,
$$

so the first map must be generically an isomorphism.

From the sequence

$$
0 \longrightarrow \mathcal{I}_{X / S} \longrightarrow \mathcal{O}_{S} \longrightarrow \mathcal{O}_{X} \longrightarrow 0
$$

one gets

$$
0 \longrightarrow \omega_{S} \longrightarrow \mathcal{H o m}_{\mathcal{O}_{S}}\left(\mathcal{I}_{X / S}, \omega_{S}\right) \longrightarrow \omega_{X} \longrightarrow 0
$$

Let

$$
\mathcal{L}=\mathcal{H o m}_{\mathcal{O}_{S}}\left(\mathcal{I}_{X / S}, \mathcal{O}_{S}\right)
$$

so

$$
\mathcal{H o m}_{\mathcal{O}_{S}}\left(\mathcal{I}_{X / S}, \omega_{S}\right)=\mathcal{L}(s-4) .
$$

We get a generic isomorphism

$$
\mathcal{L}(s-4)_{\mid D} \longrightarrow \omega_{X \mid D} \longrightarrow 0 .
$$

Dualizing, we get an isomorphism

$$
\omega_{X \mid D} \vee \mathcal{L}(s-4)_{\mid D} \vee .
$$

Let us investigate the left and right hand sides of this.

Right hand side : Since $\mathcal{L}=\mathcal{H}_{\text {om }}$ O $_{S}\left(\mathcal{I}_{X / S}, \mathcal{O}_{S}\right)$, one gets a map

$$
\mathcal{L}_{\mid D} \longrightarrow \mathcal{H o m}_{\mathcal{O}_{D}}\left(\left(\mathcal{I}_{X / S}\right)_{\mid D}, \mathcal{O}_{D}\right)
$$

giving

$$
\mathcal{H o m}_{\mathcal{O}_{D}}\left(\left(\mathcal{I}_{X / S}\right)_{\mid D}, \mathcal{O}_{D}\right)(s-4)^{\vee} \longrightarrow \mathcal{L}(s-4)_{\mid D} \vee .
$$

Left hand side: The sequence

$$
0 \longrightarrow \mathcal{F} \longrightarrow \mathcal{O}_{X} \longrightarrow \mathcal{O}_{X^{\prime}} \longrightarrow 0
$$

gives

$$
0 \longrightarrow \omega_{X^{\prime}} \longrightarrow \omega_{X} \longrightarrow \mathcal{H o m}_{\mathcal{O}_{X}}\left(\mathcal{F}, \omega_{X}\right) \longrightarrow 0 .
$$

Now by Proposition 1.1

$$
\mathcal{H o m}_{\mathcal{O}_{X}}\left(\mathcal{F}, \omega_{X}\right)=\mathcal{H o m}_{\mathcal{O}_{D}}\left(\mathcal{F}, \omega_{D}\right)
$$


This gives a generic isomorphism

$$
\omega_{X \mid D} \longrightarrow \mathcal{H o m}_{\mathcal{O}_{D}}\left(\mathcal{F}, \omega_{D}\right) \longrightarrow 0
$$

giving an isomorphism

$$
\omega_{X \mid D} \vee \cong \mathcal{H o m}_{\mathcal{O}_{D}}\left(\mathcal{H o m}_{\mathcal{O}_{D}}\left(\mathcal{F}, \omega_{D}\right), \mathcal{O}_{D}\right) .
$$

All in all, we now have

$$
\begin{aligned}
\overline{\mathcal{F}}(4-s)=\left(\mathcal{I}_{X / S} \otimes \mathcal{O}_{D}\right)^{\circ}(4-s) & \hookrightarrow \\
& \left.\longrightarrow \mathcal{I}_{X / S} \otimes \mathcal{O}_{D}\right)^{\vee \vee}(4-s) \\
& \mathcal{H o m}_{\mathcal{O}_{D}}\left(\mathcal{H o m}_{\mathcal{O}_{D}}\left(\mathcal{F}, \omega_{D}\right), \mathcal{O}_{D}\right) .
\end{aligned}
$$

We now wish to show that there is an injection

$$
\mathcal{H o m}_{\mathcal{O}_{D}}\left(\mathcal{H o m}_{\mathcal{O}_{D}}\left(\mathcal{F}, \omega_{D}\right), \mathcal{O}_{D}\right) \hookrightarrow \mathcal{H o m}_{\mathcal{O}_{D}}\left(\omega_{D}, \mathcal{F}\right) .
$$

By Proposition 1.1 there is an isomorphism

$$
\mathcal{F} \longrightarrow \mathcal{H o m}_{\mathcal{O}_{D}}\left(\mathcal{H o m}_{\mathcal{O}_{D}}\left(\mathcal{F}, \omega_{D}\right), \omega_{D}\right) \text {. }
$$

For a sheaf $\mathcal{A}$ on $D$ there is a natural map

$$
\mathcal{H o m}_{\mathcal{O}_{D}}\left(\mathcal{A}, \mathcal{O}_{D}\right) \longrightarrow \mathcal{H o m}_{\mathcal{O}_{D}}\left(\omega_{D}, \mathcal{H o m}_{\mathcal{O}_{D}}\left(\mathcal{A}, \omega_{D}\right)\right) \text {. }
$$

Putting $\mathcal{A}=\mathcal{H o m}_{\mathcal{O}_{D}}\left(\mathcal{F}, \omega_{D}\right)$ and using that (17) is an isomorphism, one gets an injection

$$
\mathcal{H o m}_{\mathcal{O}_{D}}\left(\mathcal{H o m}_{\mathcal{O}_{D}}\left(\mathcal{F}, \omega_{D}\right), \mathcal{O}_{D}\right) \hookrightarrow \mathcal{H o m}_{\mathcal{O}_{D}}\left(\omega_{D}, \mathcal{F}\right)
$$

and thus an injection

$$
\overline{\mathcal{F}} \hookrightarrow \mathcal{H o m}_{\mathcal{O}_{D}}\left(\omega_{D}, \mathcal{F}\right)(s-4)
$$

Now let

$$
\mathcal{G}=\mathcal{E} x t_{\mathcal{O}_{D}}^{2}\left(\mathcal{F}, \omega_{\mathbf{P}^{3}}\right)=\mathcal{H o m}_{\mathcal{O}_{D}}\left(\mathcal{F}, \omega_{D}\right)
$$

and correspondingly define $\overline{\mathcal{G}}$. The following is the result used to prove Theorem 2.1 .

Corollary 6.2. Suppose $D$ is a reduced and irreducible curve. If $\mathcal{G}(d)$ has a section, then $\overline{\mathcal{G}}(d+(s-2))$ has a section.

Proof. Suppose we are given a map

$$
\mathcal{F} \longrightarrow \omega_{D}(d)
$$

corresponding to a section of $\mathcal{G}(d)$. By Theorem 6.1

$$
\overline{\mathcal{F}} \subseteq \mathcal{H o m}_{\mathcal{O}_{D}}\left(\omega_{D}, \mathcal{F}\right)(s-4)
$$

We will show that we get a map

$$
\mathcal{H o m}_{\mathcal{O}_{D}}\left(\omega_{D}, \mathcal{F}\right)(s-4) \longrightarrow \omega_{D}(d+(s-2)),
$$

or, equivalently,

$$
\mathcal{H o m}_{\mathcal{O}_{D}}\left(\omega_{D}, \mathcal{F}\right) \longrightarrow \omega_{D}(d+2),
$$

giving a section of $\overline{\mathcal{G}}(d+(s-2))$. Since $H^{0} \mathcal{O}_{D}(-1)=0$, we get $H^{1} \omega_{D}(1)=0$. Thus $\omega_{D}$ is 2-regular, and hence $\omega_{D}(2)$ is generated by its sections. So we get a map $\mathcal{O}_{D} \rightarrow \omega_{D}(2)$ giving the map

$$
\mathcal{H o m}_{\mathcal{O}_{D}}\left(\omega_{D}(2), \omega_{D}(d+2)\right) \longrightarrow \mathcal{H o m}_{\mathcal{O}_{D}}\left(\mathcal{O}_{D}, \omega_{D}(d+2)\right)=\omega_{D}(d+2) .
$$


Putting this together with (18) gives

$$
\mathcal{H o m}_{\mathcal{O}_{D}}\left(\omega_{D}, \mathcal{F}\right) \longrightarrow \mathcal{H o m}_{\mathcal{O}_{D}}\left(\omega_{D}, \omega_{D}(d)\right) \longrightarrow \omega_{D}(d+2) .
$$

As a motivation for the next theorem, consider the special case where $S$ is smooth and $D$ is a smooth curve on $S$. Let $D^{(n)}$ be the multiplicity $n$ structure on $D$ in $S$. As remarked in the introduction, if $\mathcal{O}_{D^{(n)}}(-r)$ has sections for some $-r<0$, then $\omega_{D}^{\vee}(s-4)$ has positive degree. The following is a more general version, and the situation we consider is as in the first sequence in (12).

Theorem 6.3. Suppose $\mathcal{F}(-1)$ has a non-zero section $\sigma$ and let $D$ be a curve contained in the support of $\sigma$. Then $\omega_{D}(4-s)$ has no sections.

Proof. Assume to the contrary that $\omega_{D}(4-s)$ has a section. This gives an injection

$$
\mathcal{O}_{E} \hookrightarrow \omega_{D}(4-s)
$$

for some $E \subseteq D$. By Section 1 we get a sequence of torsion free modules

$$
0 \longrightarrow \hat{\mathcal{O}}_{E} \longrightarrow \omega_{D}(4-s) \longrightarrow \mathcal{A} \longrightarrow 0
$$

Dualizing once by $\mathcal{H o m}_{\mathcal{O}_{D}}\left(-, \omega_{D}\right)$, we get

$$
0 \longrightarrow \mathcal{H}_{o m_{\mathcal{O}_{D}}}\left(\mathcal{A}, \omega_{D}\right) \longrightarrow \mathcal{O}_{D}(s-4) \longrightarrow \mathcal{H o m}_{\mathcal{O}_{D}}\left(\hat{\mathcal{O}}_{E}, \omega_{D}\right) \longrightarrow 0,
$$

so $\mathcal{H o m}_{\mathcal{O}_{D}}\left(\hat{\mathcal{O}}_{E}, \omega_{D}(s-4)\right)=\mathcal{O}_{E}(s-4)$. Dualizing back, we get that (19) is the same as

$$
0 \longrightarrow \omega_{E}(4-s) \longrightarrow \omega_{D}(4-s) \longrightarrow \mathcal{A} \longrightarrow 0
$$

Thus by replacing $D$ with $E$ we may assume that a section of $\omega_{D}(4-s)$ is generically non-zero.

Next we claim that we may assume that the support of $\mathcal{F}$ is $D$. The restriction $\mathcal{F} \rightarrow\left(\mathcal{F}_{\mid D}\right)^{\circ}$ gives by push-down a diagram

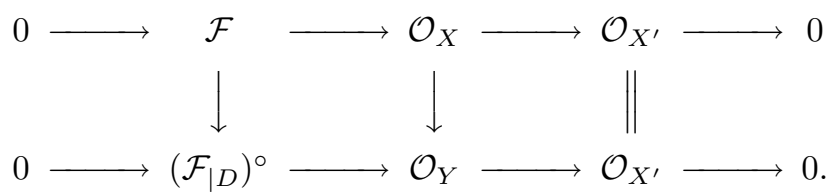

By replacing $\mathcal{F}$ by $\left(\mathcal{F}_{\mid D}\right)^{\circ}$ and $X$ by $Y$ we may then assume the claim to hold.

Now $D$ is a subscheme of $X$, so we get a composition

$$
\phi: \mathcal{O}_{D}(1) \longrightarrow \mathcal{F} \longrightarrow \mathcal{O}_{X} \longrightarrow \mathcal{O}_{D}
$$

Consider now two cases.

a. $\phi$ is zero. Then $D$ is a subscheme of $X^{\prime}$. Let $X^{\prime \prime}$ be the residual of $D$ in $X^{\prime}$, so we have a sequence

$$
0 \longrightarrow \mathcal{F}^{\prime} \longrightarrow \mathcal{O}_{X^{\prime}} \longrightarrow \mathcal{O}_{X^{\prime \prime}} \longrightarrow 0
$$

and by Theorem 6.1 we have

$$
\mathcal{F} \subseteq \mathcal{H o m}_{\mathcal{O}_{D}}\left(\omega_{D}(4-s), \mathcal{F}^{\prime}\right) .
$$

Thus if $\mathcal{F}(-1)$ has a section generically non-zero on $D$, so does $\mathcal{F}^{\prime}(-1)$. 
b. $\phi$ is non-zero. Then we get a factorization

$$
\mathcal{O}_{D}(1) \longrightarrow \mathcal{O}_{E}(1) \longrightarrow \mathcal{O}_{D}
$$

where $E$ is a proper closed subscheme of $D$. The first map is surjective and the other map is injective. Applying the dualizing functor $\mathcal{H}_{\mathrm{O}_{\mathcal{O}_{D}}}\left(-, \omega_{D}\right)$ and twisting, we get

$$
0 \longrightarrow \omega_{D}(4-s) \longrightarrow \omega_{E}(3-s) \longrightarrow \omega_{D}(3-s) \longrightarrow 0 .
$$

The composition gives a section of $\omega_{D}(3-s)$ generically non-zero on $E$. We may then proceed as in the first part of the proof and replace $D$ by $E$. Then proceed as in the second part of the proof to get $\mathcal{F}$ with support on $D$.

Now we see that in a. the degree of $X$ decreases and in b. the degree of $D$ decreases. The procedure can always be carried on. This gives a contradiciton.

Corollary 6.4. Suppose the surface $S$ is of degree three. Then the set-theoretical support of $\sigma$ is a disjoint union of lines.

Proof. Let $D$ be a reduced connected curve in the support of $\sigma$. Then $\omega_{D}(1)$ has no sections. By Hilbert polynomial considerations we see that $D$ must be a line.

Now let $D$ be a reduced curve on $S$, and $R$ another curve on $S$. The ideal $\mathcal{I}_{D}^{i} \cdot \mathcal{I}_{R}$ defines a subscheme of $S$ consisting of a locally Cohen-Macaulay curve $X^{(i)}$ and some embedded points. Let $\mathcal{F}_{i-1}$ be defined by the sequence

$$
0 \longrightarrow \mathcal{F}_{i-1} \longrightarrow \mathcal{O}_{X^{(i)}} \longrightarrow \mathcal{O}_{X^{(i-1)}} \longrightarrow 0 \text {. }
$$

Also let $D^{(i)}$ be the multiplicity $i$ structure on $D$ in $S$. So we get sequences

$$
0 \longrightarrow \mathcal{G}_{i-1} \longrightarrow \mathcal{O}_{D^{(i)}} \longrightarrow \mathcal{O}_{D^{(i-1)}} \longrightarrow 0,
$$

where $\mathcal{G}_{i-1}$ is defined as the kernel.

Let $\mathcal{J}_{i}$ be the ideal sheaf of $D^{(i)}$ in $\mathcal{O}_{S}$ and let $\mathcal{I}_{i}$ be the ideal sheaf of $X^{(i)}$ in $\mathcal{O}_{S}$. Then, as in (13),

$$
\mathcal{G}_{i}=\left(\mathcal{J}_{i} \otimes \mathcal{O}_{D}\right)^{\circ}, \quad \mathcal{F}_{i}=\left(\mathcal{I}_{i} \otimes \mathcal{O}_{D}\right)^{\circ} .
$$

From [Ba-Fo] there are natural maps

$$
\mathcal{G}_{i} \otimes \mathcal{G}_{m} \longrightarrow \mathcal{G}_{i+m}
$$

coming from the maps

$$
\mathcal{J}_{i} \cdot \mathcal{J}_{m} \longrightarrow \mathcal{J}_{i+m}
$$

Analogously there are maps

$$
\mathcal{F}_{i} \otimes \mathcal{G}_{m} \longrightarrow \mathcal{F}_{i+m}
$$

coming from the maps

$$
\mathcal{I}_{i} \cdot \mathcal{J}_{m} \longrightarrow \mathcal{I}_{i+m}
$$

Theorem 6.5. Suppose $D$ is smooth. If $\mathcal{G}_{m}=\mathcal{H o m}_{\mathcal{O}_{D}}\left(\omega_{D}, \mathcal{G}_{m-1}(s-4)\right)$, then the maps (20) and (21) are isomorphisms.

Proof. Theorem 6.1 tells us that if

$$
\mathcal{G}_{m}=\mathcal{H o m}_{\mathcal{O}_{D}}\left(\omega_{D}, \mathcal{G}_{m-1}(s-4)\right)
$$

then $\mathcal{G}_{m}=\mathcal{J}_{m} \otimes \mathcal{O}_{D}$. Since $\mathcal{G}_{m}$ is locally free on $D, \mathcal{J}_{m}$ becomes locally free on $S$. But then $\mathcal{I}_{i+m}=\mathcal{I}_{i} \otimes \mathcal{J}_{m}$ and $\mathcal{J}_{i+m}=\mathcal{J}_{i} \otimes \mathcal{J}_{m}$, so the theorem follows. 


\section{EXAMPLES ON A NON-NORMAL SURFACE}

Here we give examples showing that if $S$ is not a normal surface then Theorems 2.1 and 4.1 do not hold.

Let $S$ be an irreducible cubic surface with a double line $L$. We may assume

$$
I_{L}=(x, y) \subseteq k[x, y, z, w] .
$$

Let $L^{(2)}$ be the multiplicity 2 structure on $L$ given by the ideal

$$
\left(x^{2}, x y, y^{2}, x f+y g\right),
$$

where $f$ and $g$ are homogeneous in $z$ and $w$ of degrees $r \geq 0$ (see [Ha, Ex. 6.4). There is a sequence

$$
0 \longrightarrow \mathcal{L} \longrightarrow \mathcal{O}_{S} \longrightarrow \mathcal{O}_{L^{(2)}} \longrightarrow 0
$$

where $H_{*}^{0} \mathcal{L}$ has generators in degrees 2 and $r+1$.

According to $[\mathrm{Ha}]$, Proposition 6.6, a general map

$$
\mathcal{L}(-r) \longrightarrow \mathcal{O}_{S}
$$

gives a sequence

$$
0 \longrightarrow \mathcal{L}(-r) \longrightarrow \mathcal{O}_{S} \longrightarrow \mathcal{O}_{C} \longrightarrow 0
$$

where $C$ is a smooth curve on $S$. We then see that the generators of $I_{C}$ occur in degrees

$$
3, \quad r+2, \quad 2 r+1 .
$$

It is also easily seen that the initial ideal $\operatorname{in}\left(I_{L^{(2)}}\right)$ is represented by

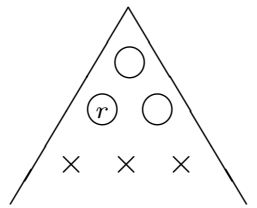

By $[\mathrm{Fl}-\mathrm{Gr}]$ the initial ideal in $\left(I_{C}\right)$ is then represented by

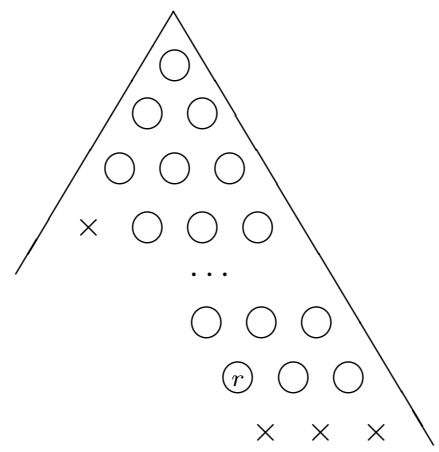


Thus we see that, disregarding the generator of degree 3 coming from a form defining the surface $S$, we have a smooth curve $C$ with a gap of $r-1$ between successive degrees of generators of in $\left(I_{C}\right)$.

\section{REFERENCES}

[Ba-Fo] C. Banica, O. Forster. Multiplicity structures on space curves. Lefschetz Centennial Conf., Part I, Contemp. Math. 58, (1986), 47-64. MR 88c:32018

[Ba-St] D. Bayer, M. Stillman. A criterion for detecting m-regularity. Inventiones Mathematicae 87 (1987), 1-11. MR 87k:13019

[Co] M. Cook. The connectedness of space curve invariants. Compositio Mathematica 111 (1998), 221-244. MR 99a:14040

[Ei] D. Eisenbud. Commutative algebra with a view towards algebraic geometry. GTM 150, Springer Verlag (1995). MR 97a:13001

[Fl] G. Fløystad. Higher initial ideals of homogeneous ideals. Memoirs of the American Mathematical Society 134 (1998), no. 638. MR 98m:13021

[Fl-Gr] G. Fløystad, M. Green. The information encoded in initial ideals. To appear in Transactions of the American Mathematical Society.

[Gi] S. Giuffrida. Graded Betti numbers and Rao module of a curve lying on a smooth cubic surface in $\mathbf{P}^{3}$. Queen's Papers in Pure and Appl. Math., 88 (1991), A1-A61. MR 93g:14035

[Ha] R. Hartshorne. Generalized divisors on Gorenstein schemes. K-theory 8 (1994), 287339. MR 95k:14008

[Li] R. Liebling. Classification of space curves using initial ideals. Ph.D. thesis, University of California at Berkeley (1996).

[MD-Pe] M. Martin-Deschamps, D. Perrin. Sur la classification des courbes gauches. Astérisque 184-185 (1990). MR 91h:14039

Matematisk Institutt, Johs. Brunsgt. 12, 5008 Bergen, Norway

E-mail address: gunnar@mi.uib.no 